\title{
Coherent Network Analysis of Gravitational Waves from Three-Dimensional Core-Collapse Supernova Models
}

\author{
Kazuhiro Hayama ${ }^{1,2}$, Takami Kuroda ${ }^{3}$, Kei Kotake ${ }^{4,5}$, and Tomoya Takiwaki ${ }^{6}$ \\ ${ }^{1}$ Department of Physics, Osaka City University, \\ Sugimoto 3-3-138, Sumiyoshi, Osaka, 558-8585, Japan \\ ${ }^{5}$ Gravitational Wave Project Office, \\ National Astronomical Observatory of Japan, \\ 2-21-1, Osawa, Mitaka, Tokyo, 181-8588, Japan \\ ${ }^{3}$ Department of Physics, University of Basel, \\ Klingelbergstrasse 82, 4056 Basel, Switzerland \\ ${ }^{4}$ Department of Applied Physics, Fukuoka University, \\ 8-19-1, Jonan, Nanakuma, Fukuoka, 814-0180, Japan \\ ${ }^{5}$ Center for Computational Astrophysics, \\ National Astronomical Observatory of Japan, 2-21-1, \\ Osawa, Mitaka, Tokyo, 181-8588, Japan and \\ ${ }^{6}$ Astrophysical Big Bang Laboratory, \\ RIKEN, Saitama, 351-0198, Japan
}




\begin{abstract}
Using predictions from three-dimensional (3D) hydrodynamics simulations of core-collapse supernovae (CCSNe), we present a coherent network analysis to detection, reconstruction, and the source localization of the gravitational-wave (GW) signals. We use the RIDGE pipeline for the analysis, in which the network of LIGO Hanford, LIGO Livingston, VIRGO, and KAGRA is considered. By combining with a GW spectrogram analysis, we show that several important hydrodynamics features in the original waveforms persist in the waveforms of the reconstructed signals. The characteristic excess in the spectrograms originates not only from rotating core-collapse, bounce and the subsequent ring down of the proto-neutron star (PNS) as previously identified, but also from the formation of magnetohydrodynamics jets and non-axisymmetric instabilities in the vicinity of the PNS. Regarding the GW signals emitted near at the rotating core bounce, the horizon distance extends up to $\sim 18 \mathrm{kpc}$ for the most rapidly rotating $3 \mathrm{D}$ model in this work. Following the rotating core bounce, the dominant source of the GW emission shifts to the non-axisymmetric instabilities. The horizon distances extend maximally up to $\sim 40 \mathrm{kpc}$ seen from the spin axis. With an increasing number of 3D models trending towards explosion recently, our results suggest that in addition to the best studied GW signals due to rotating core-collapse and bounce, the time is ripe to consider how we can do science from GWs of CCSNe much more seriously than before. Particularly the quasi-periodic signals due to the non-axisymmetric instabilities and the detectability should deserve further investigation to elucidate the inner-working of the rapidly rotating CCSNe.
\end{abstract}




\section{INTRODUCTION}

Significant progress has been made in the development of an international network of gravitational wave (GW) detectors. Although the first detection has not been accomplished yet, the non-detection has already yielded scientific results setting upper bounds to a rich variety of astrophysical GW sources (e.g., [1 6] $)$. The second generation detectors such as Advanced LIGO [7], Advanced VIRGO [8], and KAGRA [9, 10], will be on line in the coming years. The possibility to construct the third generation detectors is also recently being proposed [11, 12]. At such a high level of precision, these advanced detectors are enough sensitive to many compact objects, including binary neutron star (black hole) systems (e.g., [13-15]), neutron star normal mode oscillations (e.g., [16]), rotating neutron star mountains (e.g., [17]), and core-collapse supernova (CCSN) explosions (e.g., [18-20] for recent reviews), on the final of which we focus in this work.

According to the Einstein's theory of general relativity (e.g., [21]), no GWs can be emitted if gravitational collapse of the stellar core proceeds perfectly spherically symmetric. To produce GWs, the gravitational collapse should proceed aspherically and dynamically. Gathered over the last decades, observational evidence from electromagnetic-wave observations, e.g., of ejecta morphologies, spatial distributions of nucleosynthetic yields (as recently discovered by the NuSTAR observations of Cas A [22]) and natal kick of pulsars has pointed towards CCSNe indeed being generally aspherical (i.e., multi-dimensional (multi-D), e.g., [23 26] and references therein). Unfortunately, however, these electromagnetic signatures are rather indirect to probe the inner-working because they can only provide an image of optically thin regions far away from the central core.

Much more direct information is carried away by neutrinos and GWs. The detection of neutrinos from SN1987A paved the way for the neutrino astronomy [27, 28]. Even though there were just two dozen neutrino events from SN1987A (which are not enough to say something solid about the multi-D feature), these events have been studied extensively (yielding $\sim 500$ papers) and have allowed us to have a confidence that our basic picture of the supernova physics is correct (e.g., [29], see [30] for a recent review). In propagating the stellar envelope, SN neutrinos produced deep inside the core are influenced (at least) by the well-known Mikheyev-Smirnov-Wolfenstein effect (e.g., [31, 32]). Therefore, GWs are primary observables, which carry us a direct episode of the supernova engine. 
From a theoretical point of view, clarifying what makes the dynamics of the central engine deviate from spherical symmetry is essential also in understanding the yet uncertain CCSN mechanism. As a result of continuing efforts for decades, theory and neutrino radiationhydrodynamics simulations are now converging to a point that multi-D hydrodynamic matter motions play a crucial role in facilitating the neutrino mechanism, which is the most favoured scenario to trigger explosions (e.g., [18, 33 35] for recent reviews). The neutrino mechanism [36, 37] requires convection and the standing-accretion-shock instability (SASI) to increase the neutrino heating efficiency in the gain region where net energy absorption is positive. For canonical massive stars heavier than $\sim 10 M_{\odot}$, the neutrino mechanism fails in spherical symmetry (1D) [38 41]. A number of two-dimensional (2D) simulations with spectral neutrino transport now report successful neutrino-driven models that are trending towards explosion [42 45], whereas the first such three-dimensional (3D) simulations [46 48] have reported explosions only for a light progenitor model.

Another candidate mechanism is the magnetohydrodynamic (MHD) mechanism [49 54]. Rapid rotation of precollapse iron cores is preconditioned for this mechanism, because it relies on the extraction of rotational free energy of the core by means of the field-wrapping and magnetorotational instability (e.g., [55 57] and references therein). Such rapid rotation is likely to obtain $\sim 1 \%$ of massive star population (e.g., [58]). Minor as they may be, the MHD explosions are receiving great attention as a possible relevance to magnetars and collapsars (e.g., [59 62]), which are hypothetically linked to the formation of long-duration gamma-ray bursts (e.g., 63] for a review).

Keeping step with these advances in the CCSN theory and modelling, considerable progress in understanding the GW emission processes has been made simultaneously (e.g., [19, 20, 64] for recent reviews). In the MHD mechanism, rapid rotation of the precollapse core leads to significant rotational flattening of the collapsing and bouncing core, leading to a theoretically best-studied, the so-called type I waveform of the bounce signals. The waveform is characterized by sharp spikes at bounce followed by a subsequent ring-down phase [65 67]. After bounce, a large variety of the emission processes have been proposed, including convective motions in the proto-neutron star (PNS) and in the region behind the stalled shock [68 71], the SASI (e.g., [72 76]), non-axisymmetric rotational instabilities [77, 80], anisotropy in neutrino emission [71, 74, 81, 82], and pulsations of the PNS [83].

If we were able to associate the above GW signatures with the proposed explosion mecha- 
nisms (basically either the neutrino or MHD mechanism), then the GW signals, if successfully detected, should help confirm the mechanisms. To this end, one must extract a real GW signal buried in detector noises and determine the waveform characters by matching somehow to signal predictions obtained from the multi-D CCSN simulations mentioned above.

The most established method is matched filtering (see [13] for review) as is done when looking for compact binary coalescence signals (e.g., [84]). However, such a template based search is not suitable for the GW signals from CCSNe. This is because the waveforms, except for the bounce signals in rapidly rotating cores, are all affected by turbulence in the postbounce phase, which is governed by the non-linear hydrodynamics (e.g., [19, 64]). Hence, the waveforms are of stochastic nature [74] and impossible to predict a priori. To detect such signals from the next nearby event and extract the information of the explosion physics, one needs to construct a suitable analysis method to signal extraction, reconstruction, and model selection, which is able to deal with the stochastic GW nature.

Considering GW signals from CCSN simulations into signal detection/reconstruction and parameter estimation (of the supernova physics) was pioneered by Brady and Ray-Majumder [85]. The authors introduced a Gram-Schmidt method to parameterize the bounce GW signals [86] using a small set of orthonormal basis vectors that represent characteristic features common to all the waveforms. More efficient method to derive the basis vectors was introduced by [87] with principal component analysis (PCA). Röver et al. [88] combined the PCA with the Bayesian inference to recover the bounce GW signals by [89] and obtained excellent waveform reconstructions. Going step a further, Logue et al. [90] developed a Bayesian model selection framework to tell the proposed explosion mechanisms (MHD, neutrino, or acoustic mechanism) apart in the presence of detector noises. They pointed out that the Bayesian approach could identify any of the candidate mechanisms with high confidence for CCSN events at distances of up to $\sim 2 \mathrm{kpc}$.

The above work has demonstrated that the PCA is indeed a powerful tool to extract robust waveform features of the bounce signals in rapidly rotating core-collapse. However as already mentioned by [87, 88, 90, 91], one of the disadvantage is that only from the PCs it is not easy to directly extract the physical parameters of the central core (such as the rotational parameters in this case). To get around the difficulty, Engels et al. [91] recently presented a multivariate regression model, by which several important parameters to determine the bounce signals (i.e., in the context of the MHD mechanism) were shown to 
be nicely extractable. Yet, as the authors mentioned, their current regression model cannot deal with the stochastic waveforms, which are inherent to the neutrino mechanism, probably the most canonical way to blow up massive stars.

There have been other approaches to detection and reconstruction of the GW signals from stellar core collapse. The discipline stems from the work by Gürsel and Tinto [92], in which a maximum likelihood approach was introduced to reconstruct the time evolution of the burst GW signals. More efficient methods for inferring the incident GW signals have been proposed so far including the Tikhonov regularization scheme by [93, 94] and a maximum entropy approach [95]. Summerscales et al. [95] successfully reconstructed the injected waveforms by [96] using data from two detectors without any a priori knowledge of the signal shape. Extending the Tikhonov regularization of [93], Hayama et al. 994] added new time domain data conditioning to the analysis pipeline, creating complete standalone coherent network analysis pipeline called RIDGE. Using the pipeline, they explored the possibility whether one could infer the degree of differential rotation from a small set of waveforms by [97], which is more recently reexamined by [98] with a more complete set of waveforms.

Joining in these efforts, we present a coherent network analysis for detection and waveform reconstruction of the GW predictions obtained from our 3D CCSN simulations. The network we consider in this work consists of the $4 \mathrm{~km}$ LIGO Hanford (H), LIGO Livingston (L), VIRGO (V), and KAGRA (K) interferometers [8, 10, 99]. One of the advantage using such world-wide detector networks is that both of the GW polarisations $\left(h_{+}\right.$and $\left.h_{\times}\right)$can be reconstructed, furthermore permitting the source position on the sky map to be determined. In most of the work mentioned above, the employed CCSN models are limited to 2D, which can produce only linearly polarized signals, and a single detector has been often considered for simplicity [88, 90]. By performing Monte Carlo simulations using the RIDGE pipeline [94], we discuss detectability of the gravitational waveforms from our 3D models and discuss to what extent information about the CCSN engine could be extracted from successful GW detection of the future nearby CCSN event.

This paper is structured as follows. In Section II, after we shortly review the candidate CCSN mechanisms, we summarize the individual gravitational waveforms that we employ in this work. Then we discuss the detectability of the GW signals in a most prevalent way, that is, by comparing the root-sum-square (rss) waveform amplitudes with the sensitivity curves 
of various GW interferometer detectors. Main results of this work are given and discussed in Section III. We summarize our results and discuss their implications in Section V.

\section{GRAVITATIONAL-WAVE SIGNATURES AND THEIR OPTIMISTIC DE- TECTABILITY}

In this study, we consider the neutrino mechanism and the MHD mechanism for CCSN explosions and describe their characteristic GW signatures in the following sections. Regarding the neutrino mechanism, we take the model waveforms from 3D simulations by Kotake et al. [74, 100], which we refer to them as $\mathrm{KK}+09$ and $\mathrm{KK}+11$ waveforms (e.g., top panel in Figure 1), respectively. For the waveforms in the context of the MHD mechanism, we use the waveforms from 3D models by Kuroda et al. [80] (e.g., middle panels in Figure 1) and 2D models by Takiwaki and Kotake [54] (e.g., bottom panels in Figure 1) (KTK14 and TK11 for short below, respectively). In Appendix A, the waveform properties of the $\mathrm{KK}+09, \mathrm{KK}+11$, KTK14, and TK11 catalogues are summarized with the numerical methods and initial conditions, respectively. Validities and variations of the model waveforms are discussed elsewhere in the following.

\section{A. Model Predictions versus Sensitivity Curves}

\section{Characteristic frequency and root-sum-square of $G W$ signals}

Figure 2 shows the (frequency-integrated) root-sum-square (rss) strain amplitude $\left(h_{\mathrm{rss}}\right)$ from the $\mathrm{KK}+$, KTK14, and TK11 catalogues against the characteristic frequency $\left(f_{c}\right)$ relative to the sensitivity curves of KAGRA, advanced LIGO (labelled as aLIGO), and advanced VIGRO (labelled as advVirgo). The sources are assumed to be located at $10 \mathrm{kpc}$ from the Earth and optimally oriented to the design sensitivity of the detectors. Following [117], we calculate the spectral density $h_{\mathrm{rss}}\left[\mathrm{Hz}^{-1 / 2}\right]$ from the Fourier transform of the wave signals $\left(h_{+, \times}(f)=\int_{-\infty}^{\infty} e^{2 \pi i f t} h_{+, \times}(t) d t\right)$ as $h_{\mathrm{rss}}=\sqrt{\sum_{A=+, \times}\left|\tilde{h}_{A}(f)\right|^{2}}$, and the (detector-dependent) characteristic frequency as,

$$
f_{c}=\left(\int_{0}^{\infty} \frac{\sum_{A} \tilde{h}_{A}(f) \tilde{h}_{A}^{*}(f)}{S_{\mathrm{n}}(f)} f \mathrm{~d} f\right) /\left(\int_{0}^{\infty} \frac{\sum_{A} \tilde{h}_{A}(f) \tilde{h}_{A}^{*}(f)}{S_{\mathrm{n}}(f)} \mathrm{d} f\right),
$$



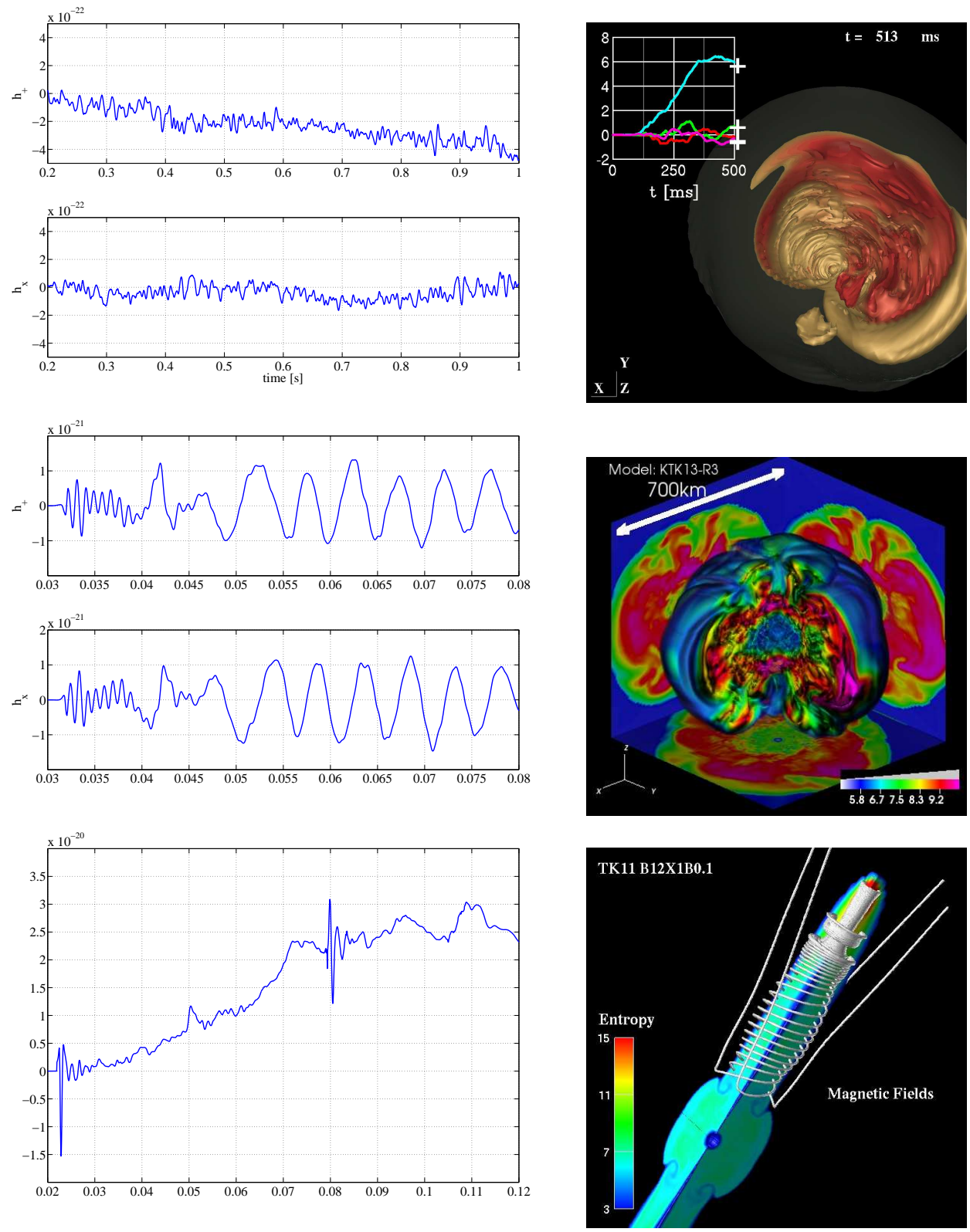

FIG. 1. GW signal predictions (left panels) for a Galactic event (at a distance of $10 \mathrm{kpc}$ ) and the blast morphologies (right panels) for the neutrino mechanism (top panels from $\mathrm{KK}+11$ ) and the 3D general-relativistic (GR) models that exhibit non-axisymmetric rotational instability (middle panels from KTK14) and jet-like explosion (bottom panels from TK11) possibly associated with the MHD mechanism. All the waveforms (left panels) come from quadrupole matter motions, whereas the inset of the top right panel shows the waveform only from anisotropic neutrino emission (see Appendix A for more details). Not to make the plots messy, waveforms seen only from the polar direction (with respect to the computational domain) are shown for the 3D models (top left and middle left panels) and waveforms seen only from the equatorial plane for the $2 \mathrm{D}$ model (bottom left panel). The time $\left(t_{\text {sim }}\right)$ is measured from the epgch when simulations are started. The polarization of the GWs is indicated by "+" and " $\times "$ (see Appendix A for more details). 


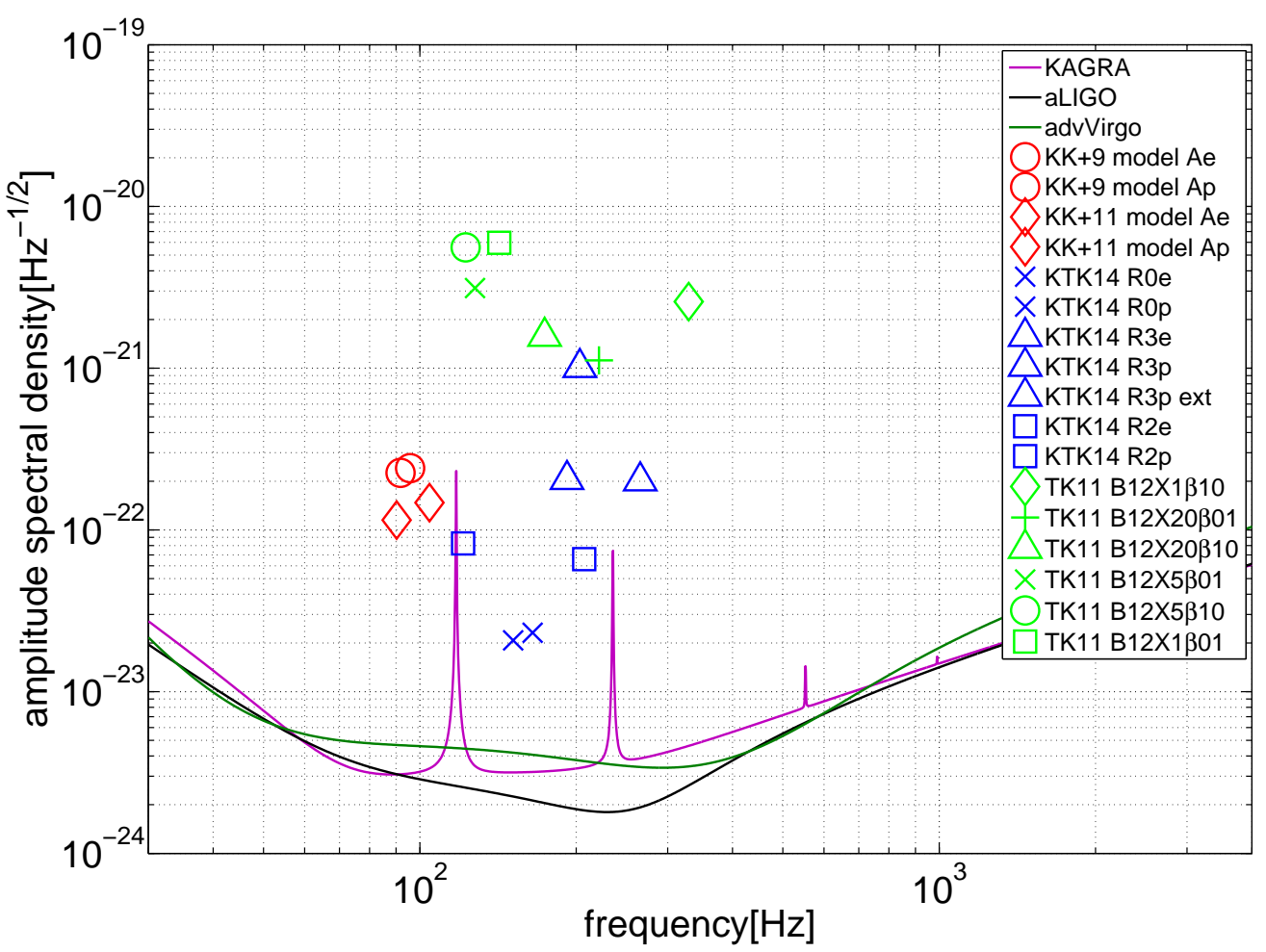

FIG. 2. Location of GW signal predictions for all the waveform catalogues of KK+ (circles and diamonds colored by red), KTK14 (circles, triangles, cross, and squares colored by blue), and TK11 (triangles, squares, crosses colored by green) in the $h_{\mathrm{rss}^{-}} f_{c}$ plane relative to the sensitivity curves of KAGRA, advanced LIGO (labelled as aLIGO), and advanced VIGRO (labelled as advVirgo). The source is located at a distance of $10 \mathrm{kpc}$.

where $S_{\mathrm{n}}(f)$ is the detector noise power spectral density in units of $\mathrm{Hz}^{-1 / 2}$. Having the same unit as the strain equivalent spectrum density, $h_{\mathrm{rss}}$ is often used in burst GW searches to compare the signal strength with the detector sensitivity.

In the $h_{\mathrm{rss}}-f_{\mathrm{c}}$ plane (Figure 2), the KK+ catalogues (symbols colored by red) that can be associated with the neutrino mechanism are rather localized to a small region (e.g., $f_{\mathrm{c}} \sim 100 \mathrm{~Hz}$ and $\left.h_{\mathrm{rss}} \sim 1-2 \times 10^{-22}\left[\mathrm{~Hz}^{-1 / 2}\right]\right)$ for the $3 \mathrm{D}$ models without or with rotation (labelled as $\mathrm{KK}+09$ or $\mathrm{KK}+11$ ) either seen from equator or pole (with the model name ending with e or $\mathrm{p}$, such as $\mathrm{KK}+09 \mathrm{Ae}$ or $\mathrm{KK}+09 \mathrm{Ap})$. This is because the initial rotation rate assumed in the $\mathrm{KK}+11$ models is not enough rapid, as is consistent with outcomes of recent stellar evolutionary calculations [102, to affect the quadrupole matter motions in the postshock region. 
Regarding the waveforms that can be associated with the MHD mechanism, the KTK14 models (symbols colored by blue) and the TK11 models (symbols colored by green) are in the range of $f_{c}=100-300 \mathrm{~Hz}, h_{\mathrm{rss}} \sim 2-100 \times 10^{-23}\left[\mathrm{~Hz}^{-1 / 2}\right]$ and of $100-300 \mathrm{~Hz}, h_{\mathrm{rss}} \sim$ $1-6 \times 10^{-21}\left[\mathrm{~Hz}^{-1 / 2}\right]$, respectively.

For the KTK14 models, it can be seen that the rss amplitudes become higher for models with larger initial angular momentum (model R3 (blue triangle) followed in order by R2, R1 (not shown in the plot), and R0 (non-rotating)). Note that for model "R3p ext" we manually extrapolate the quasi-periodic gravitational waveform (middle panel in Figure 1 ) up to $1 \mathrm{~s}$ after bounce, assuming that the non-axisymmetric instability observed in the limited simulation time (until $\sim 60 \mathrm{~ms}$ after bounce) persists afterwards (with the mean oscillation period during the simulation time) up to $1 \mathrm{~s}$ after bounce. This ad-hoc model has the maximum amplitude among the KTK14 catalogue (the highest amplitude among the blue triangles) [119].

The wave amplitudes for TK11 are generally higher than those for KTK14 simply because the assumed initial rotation rates are generally higher for the TK11 catalogue. As is well known from previous studies (e.g., [19]), the characteristic frequency $\left(f_{c}\right)$ becomes generally lower for models with larger initial angular momentum (e.g., compare R3 with R2 and $\mathrm{B} 12 \mathrm{X} 1 \beta 10$ with $\mathrm{B} 12 \mathrm{X} 20 \beta 01)$. This is because the central density $\left(\rho_{c}\right)$ in the vicinity of PNS becomes generally lower for models with larger initial angular momentum due to the stronger centrifugal forces. This makes the dynamical timescale $t_{\text {dyn }} \sim\left(G \rho_{c}\right)^{-1 / 2}$ (with $G$ being the gravitational constant) longer and the typical frequency $\left(f_{c} \propto 1 / t_{\mathrm{dyn}}\right)$ lower.

As can be seen from Figure 2, the signal predictions taken in this work are above the sensitivity curves of the advanced detectors for a Galactic event. And it is also worth mentioning that the characteristic frequency for all the models is in the range of 100 $400 \mathrm{~Hz}$, which is close to the highest sensitivity domain of the advanced detectors. In the

next section, we proceed to discuss the detectability more quantitatively by calculating the Signal-to-Noise Ratio (SNR).

\section{Optimal horizon distances for KAGRA}

Before presenting a multiple detector analysis from the next sections, we briefly compute matched-filtering SNR in this section. By taking KAGRA as an example detector and 
TABLE I. Optimal SNR as a function of distance to the source for several representative models for GW emission in the context of the MHD mechanism (KTK14 and TK11) and the neutrino mechanism $(\mathrm{KK}+)$. Theoretical noise power spectral density for KAGRA is used. As a threshold to claim detection, we take the SNR of 8 .

\begin{tabular}{|c|c|c|}
\hline Model & SNR at $10 \mathrm{kpc}$ & Distance at $\mathrm{SNR}=8[\mathrm{kpc}]$ \\
\hline KTK14 R0e & 7.35 & 9.1875 \\
\hline KTK14 R0p & 7.55 & 9.4375 \\
\hline KTK14 R2e & 21.62 & 27.0250 \\
\hline KTK14 R2p & 22.88 & 28.6 \\
\hline KTK14 R3e & 58.65 & 73.3125 \\
\hline KTK14 R3p & 73.93 & 92.4125 \\
\hline KTK14 R3p ext & 360.97 & 451.2125 \\
\hline TK11 B12X1 $\beta 10$ & 386.54 & 483.175 \\
\hline TK11 B12X20 $\beta 01$ & 78.38 & 97.975 \\
\hline TK11 B12X20 $\beta 10$ & 191.75 & 239.6875 \\
\hline TK11 B12X5ß01 & 248.63 & 310.7875 \\
\hline TK11 B12X5 $\beta 10$ & 306.2 & 382.75 \\
\hline TK11 B12X1 $\beta 01$ & 327.64 & 409.55 \\
\hline $\mathrm{KK}+9 \mathrm{Ae}$ & 13.88 & 17.35 \\
\hline $\mathrm{KK}+9 \mathrm{Ap}$ & 13.28 & 16.6 \\
\hline $\mathrm{KK}+11 \mathrm{Ae}$ & 9.43 & 11.7875 \\
\hline $\mathrm{KK}+11 \mathrm{Ap}$ & 10.38 & 12.975 \\
\hline
\end{tabular}

assuming perfect orientation, we compute the SNR as SNR $=\frac{h_{c}}{\sqrt{f_{c} S_{\mathrm{n}}\left(f_{c}\right)}}$, where $f_{c}$ is the characteristic frequency (equation (1)) and $h_{c}$ is the characteristic strain amplitude [118],

$$
h_{c} \equiv\left(3 \int_{0}^{\infty} \frac{S_{\mathrm{n}}\left(f_{c}\right)}{S_{\mathrm{n}}(f)} \sum_{A} \tilde{h}_{A}(f) \tilde{h}_{A}^{*}(f) f \mathrm{~d} f\right)^{1 / 2}
$$

For computing the SNR, we employ the theoretical noise spectral densities $S_{\mathrm{n}}$ of KAGRA from $[?]$.

Table I summarizes the optimal SNR at a distance of $10 \mathrm{kpc}$ and the distance at which 
the SNR is at 8 for the representative models, respectively. To claim detection, a SNR significantly greater than unity and probably in the range of 8 - 13 would be needed [13, 117]. We optimistically take the threshold as 8 , by which the so-called horizon distance is conventionally defined, which is the maximum distance at which the GW signals from an optimally oriented and optimally located source could be detected. It should be also mentioned that the detection distances with realistic noise (rather than idealised Gaussian noise considered here) can be significantly worse, which remains to be investigated more in detail.

The horizon distance of the KTK14 waveforms is in the range of 9 - $450 \mathrm{kpc}$ and it becomes longer for models with larger initial angular momentum and longest $\sim 450 \mathrm{kpc}$ for the extrapolated waveform (model "R3p ext"). For the non-rotating (R0) model, the detection distance is much the same either seen from pole (model R0p) or equator (model R0e) (with respect to the source coordinate system), both of which closely reach to $\lesssim 10 \mathrm{kpc}$ for the SNR $=8$ threshold. As one would guess easily, the pole to equator asymmetry in the horizon distance becomes remarkable for models with larger initial angular momentum (compare model R3p with R3e. Seen from the polar direction (e.g., parallel to the rotational axis), the SNR becomes generally higher because the more efficient GW emission is associated with the violent growth of the non-axisymmetric instabilities in the KTK14 models.

For 2D models that produce explosions by the MHD mechanism. the detection distance extends from $\sim 100$ to $\sim 480 \mathrm{kpc}$, depending on the initial rotation rates (bigger for more rapidly rotating models). These TK11 models provide the most distant horizons in this work. Due to the absence of such rapid rotation, the detection distance becomes much smaller (12 - $17 \mathrm{kpc}$ ) for the $\mathrm{KK}+$ models. As one would expect, the pole to equator asymmetry in the horizon distance is only weak in the $\mathrm{KK}+$ waveforms.

Having discussed the SNR and the optimistic detectability with a very idealized situation (i.e., a single detector for an optimally oriented and optimally located source), we shall turn to a more realistic situation, in which multiple detectors are used for an arbitrary oriented source. 


\section{RESULTS}

In section II, we have discussed an ideal detection limit with the design sensitivity of KAGRA. In reality the performance of the detection strongly depends on the antenna pattern functions of a network of the multiple detectors. In this section we study the performance using the RIDGE pipeline which takes full advantage of the global network of currently working and future interferometers (LIGO Hanford (H), LIGO Livingston (V), VIRGO (V), and KAGRA $(\mathrm{K})$ ), resulting in enhanced detection efficiency (see Appendix B and [94, 125] for more details).
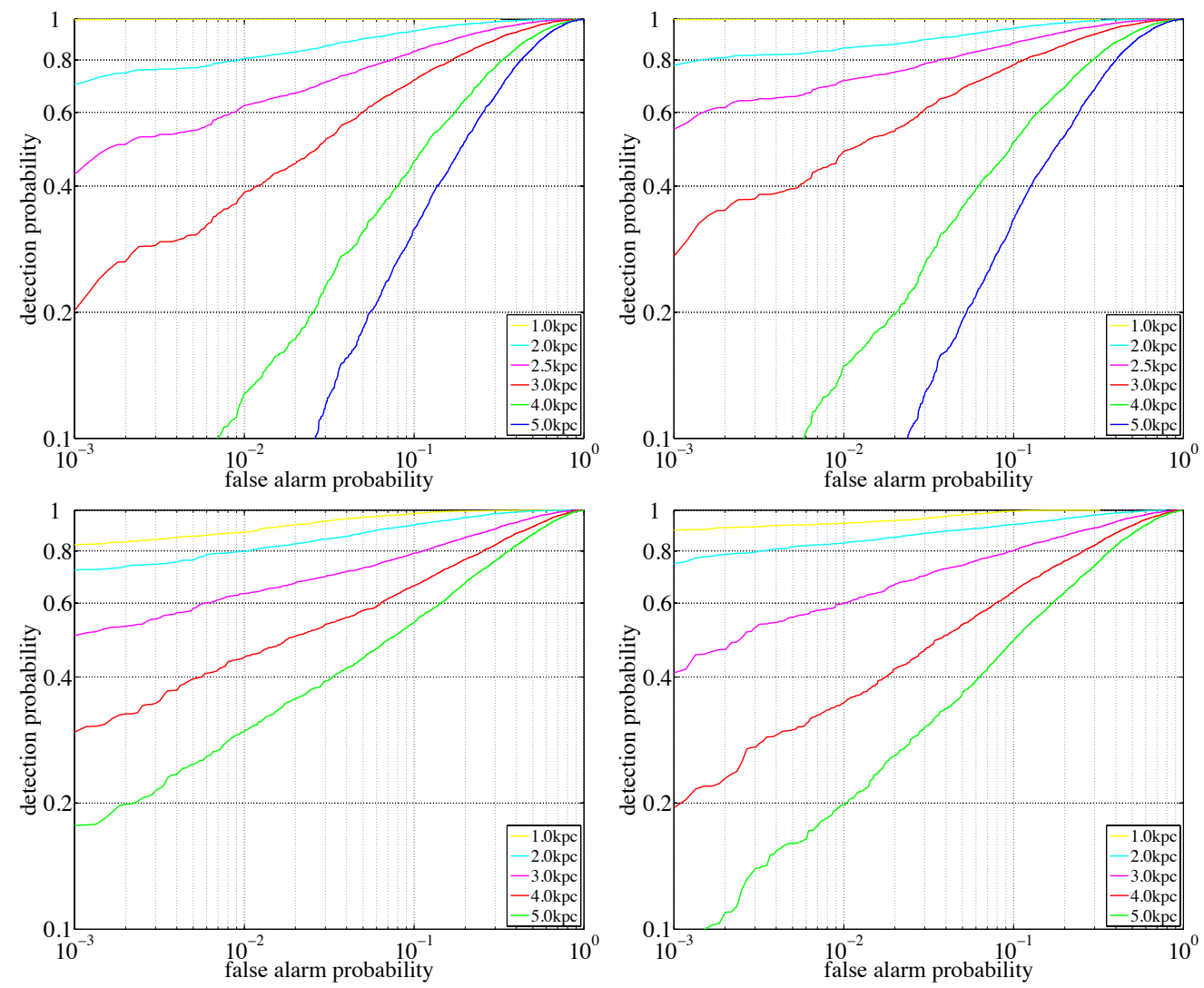

FIG. 3. ROC curves of non-rotating 3D models from the KK+09 (top panels) and KTK14 catalogues (bottom panels) for polar (left panels) and equatorial (right panels) observer at different source distances. 


\section{A. Detection efficiency}

We first focus on the receiver operating characteristic (ROC) curves, which are useful to see how the detection efficiency ( $y$ axis) changes with the false alarm probability ( $x$ axis) for sources at different distances.

Figure 3 is for the non-rotating 3D models from the KK+09 (top panels) and KTK14 catalogues (bottom panels). Without rotation, the detection efficiency is much the same either seen from pole (left panels) or equator (right panels). If we set the false alarm probability of 0.01 and the detection probability of 0.5 as a threshold of the detection, the detection distance of the non-rotating model is $2.5-3 \mathrm{kpc}$ from the $\mathrm{KK}+09$ catalogue and $\sim 3-4 \mathrm{kpc}$ from the KTK14 catalogue, respectively. It is noted that the detection horizon becomes smaller by a factor of $\sim 5$ compared to that for the most optimal situation (shown in Table I]).

Figure 4 is for 3D models with rapid rotation (i.e., the KTK14 catalogue). As already mentioned in Appendix A, the growth of non-axisymmetric instabilities was clearly observed in model R3 (the most rapidly models in KTK14, top panels in Figure 4) in the vicinity of the equatorial plane. Due to this, the horizon distance of model R3 (top panels) is bigger seen from pole $(\sim 60 \mathrm{kpc}$, left panel) compared to that seen from equator $(\sim 25 \mathrm{kpc}$, right panel). As similar to the non-rotating models (Figure 3), the horizon distances for the rapidly rotating models become up to a factor of $\sim 3$ smaller compared to the most optimal case (e.g., Table 3, $92 \mathrm{kpc}$ for pole and $\sim 73 \mathrm{kpc}$ for equator for model R3). Figure 5 shows the horizon distance of our 2D MHD model (B12X1 $\beta 10$, TK11) which has the biggest optimistic SNR in our catalogues. The horizon distance changes from the optimal estimation of $\sim 483 \mathrm{kpc}$ (Table 3 ) to $\sim 150 \mathrm{kpc}$ in the more realistic situation. These 2D models as well as the 3D rapidly rotating models have pronounced GW peaks either associated with core bounce or with the non-axisymmetric instabilities. As a result, the reduction in the horizon distance relative to the most idealized situation becomes smaller than for the non-rotating models. 

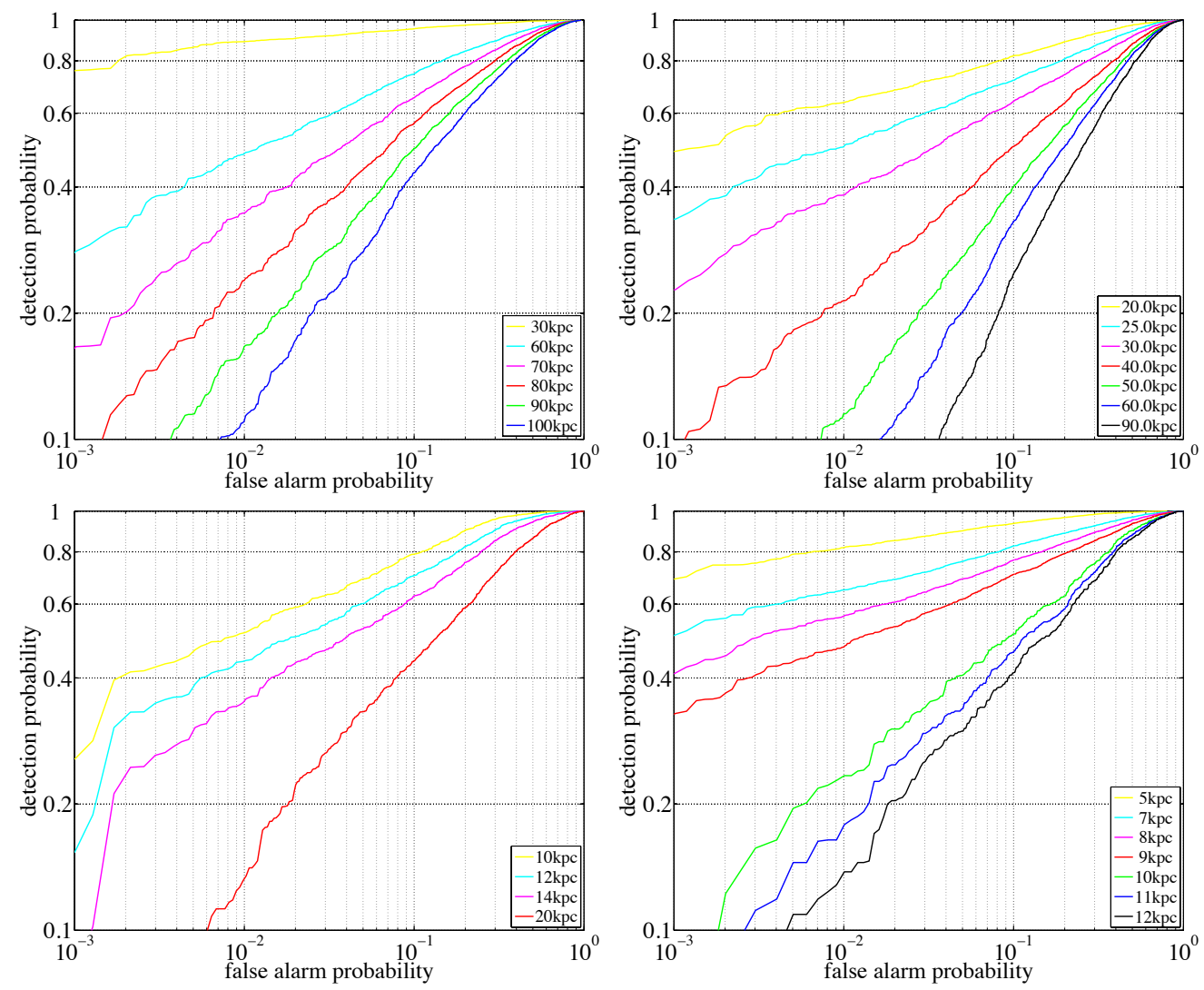

FIG. 4. Same as Figure 3 but for 3D models with rotation from the KTK14 waveforms (model R3 (top panels) and R2 (bottom panels).

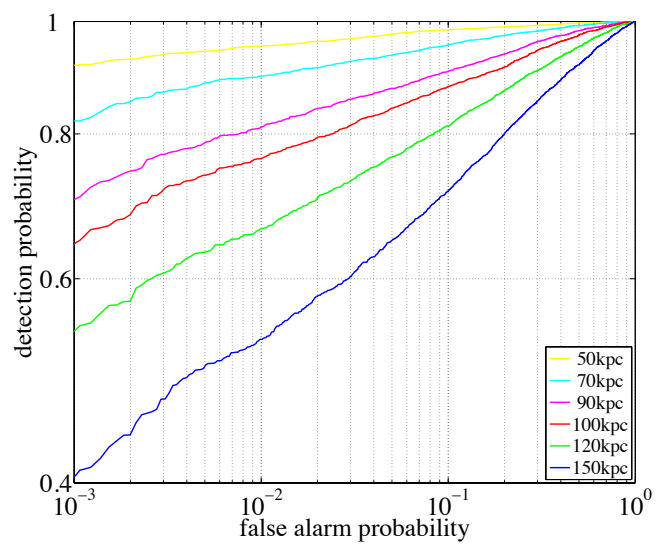

FIG. 5. Same as Figure 3 but for the 2D MHD model (B12X1 $\beta 10)$ of the TK11 waveform that possesses the biggest SNR (e.g., Table 1) in our catalogue. 

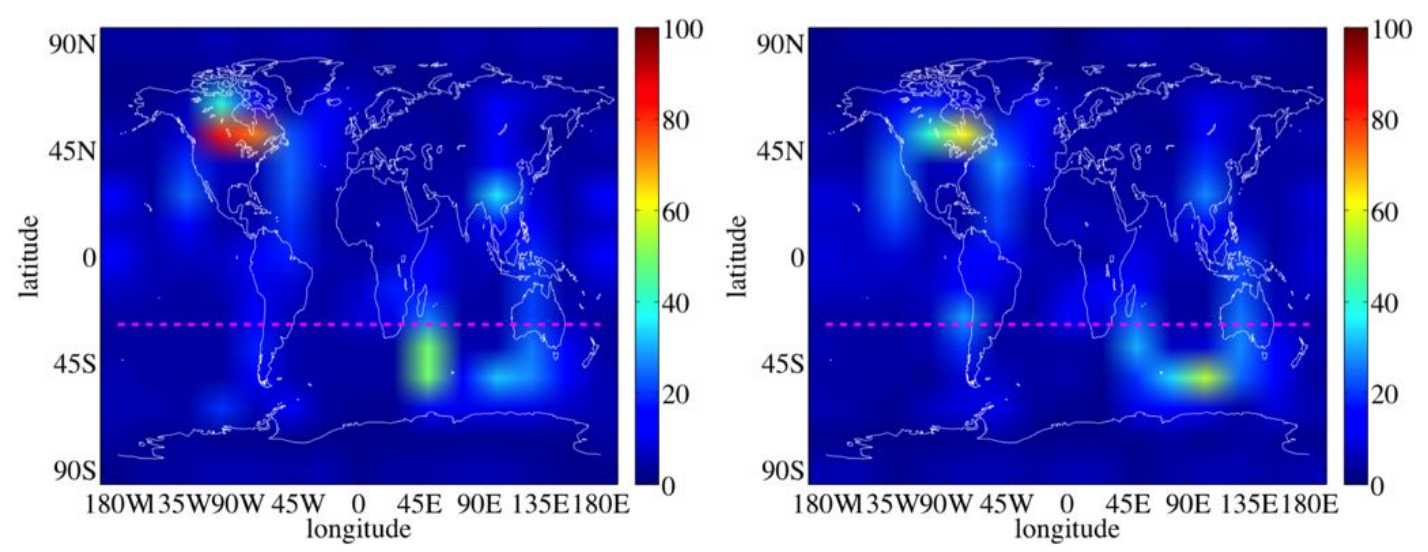

FIG. 6. Plots of the skymap from the network analysis of H-L-V-K for the 3D non-rotating (top panels) from the $\mathrm{KK}+09$ waveform for the equatorial (left) or polar (right) observer, respectively. The horizontal yellow line corresponds to the dairy motion of the Galactic center in the skymap. Note that for the advanced detectors such as advanced LIGO, advanced Virgo and KAGRA, the Galactic Center is a sky direction with high probability of the detection of GWs from the CCSNe. The $x$ and $y$ axis is the longitude and latitude, respectively. Note in this panel that the distance to the source is set as $2 \mathrm{kpc}$ (see text for more details).

\section{B. Position reconstruction}

Based on the Monte Carlo simulations using the RIDGE (e.g., Appendix B), we discuss the signal reconstruction of the sky location in this section.

Following the method in [123, 131], we inject all our model waveforms onto the simulated detector data streams (with the Gaussian noise) in a wide range of SNRs with the coordinates uniformly distributed in the sky. These signals are injected 20 times every $3 \mathrm{~s}, 0.2 \mathrm{~s}$, and 1 s for the KK+, KTK14, and TK11 waveforms, respectively. The duration of each injection is different, reflecting the different simulation timescale. For each injected event, the skymap $(\boldsymbol{S}(\theta, \phi)$, Appendix B) is calculated with the angular resolution of $d \Omega=4 \times 4$ square degrees. To quantify the accuracy of the skymap localization for a single injection, we calculate an error region: total area of all pixels in the sky which satisfy the condition $\boldsymbol{S}(\theta, \phi) \geq \boldsymbol{S}\left(\theta_{i}, \phi_{i}\right)$, where $\boldsymbol{S}\left(\theta_{i}, \phi_{i}\right)$ is the injection sky location. In the following, we choose a threshold of $50 \%$ (which contains $50 \%$ of injections) to estimate the error regions (namely, we take the $50 \%$ CL error regions). 

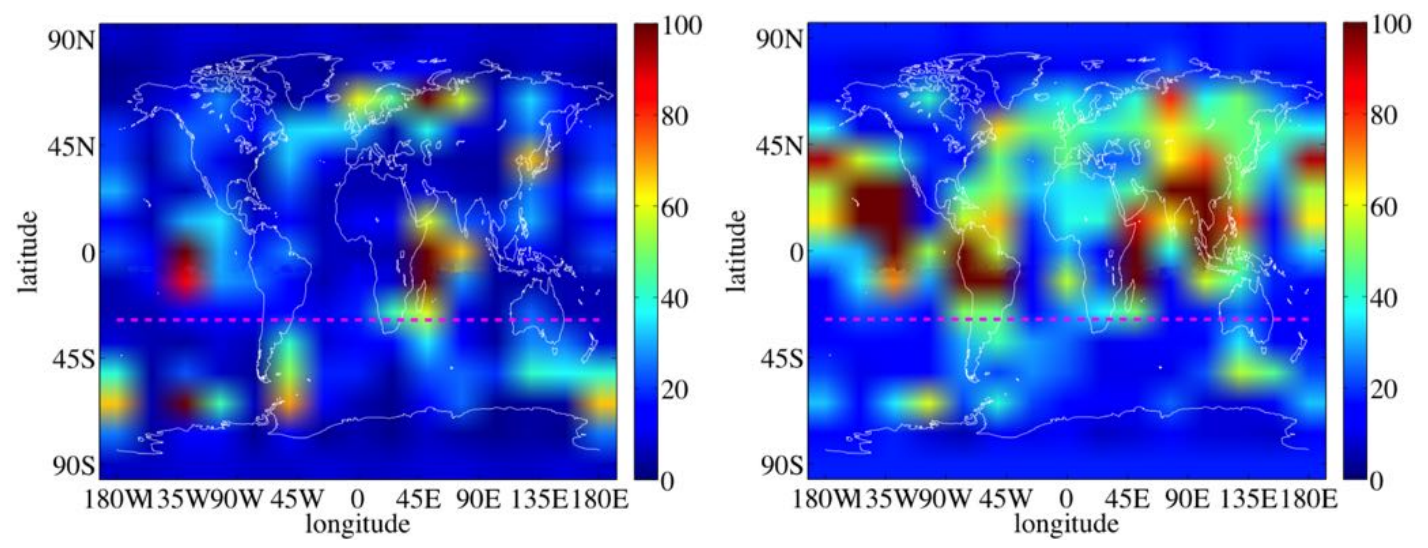

FIG. 7. Same as Figure 6 but for 3D models with rapid rotation from the KTK14 waveforms (model R3) either seen from equator (left) or from pole (right). Note in this panel that the distance to the source is set as $10 \mathrm{kpc}$.
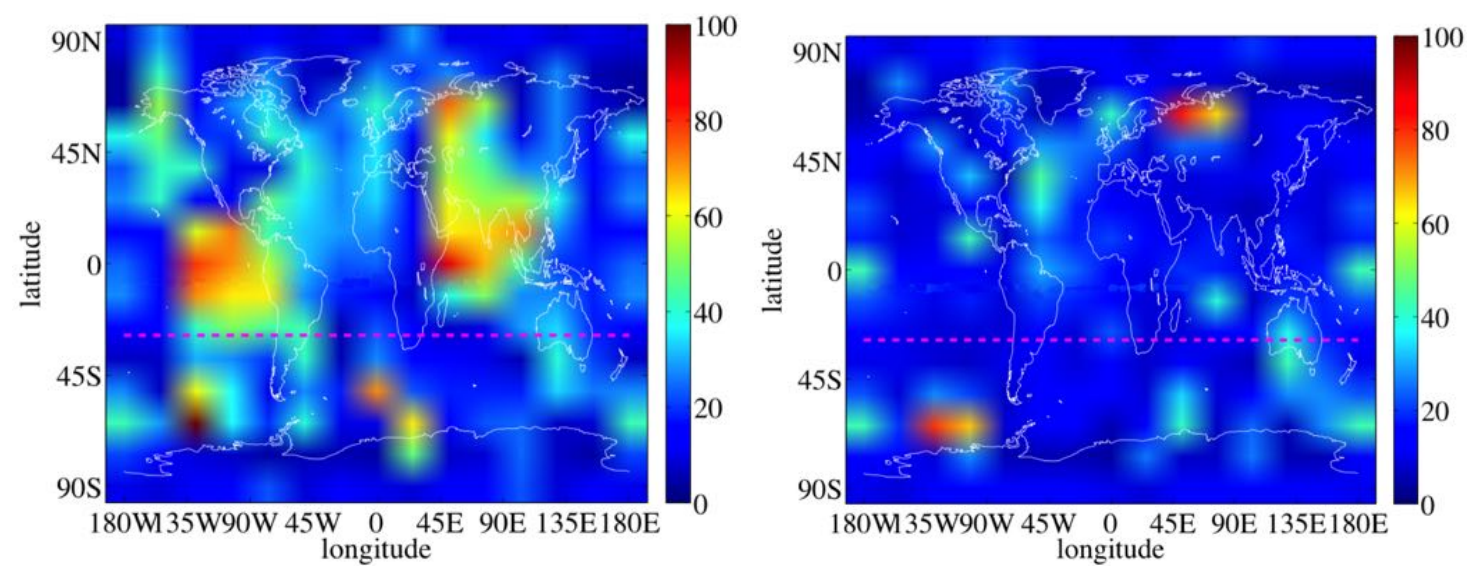

FIG. 8. Same as Figure6 but for 2D MHD models of the TK11 waveforms (models B12X5 $\beta 01$ (left panel) and B12X5 310 (right panel). Note in this panel that the distance to the source is set as 50 kpc.

Figures 6, 7, and 8 show the distributions of the error regions as a function of latitude $(\theta)$ and longitude $(\phi)$ for the KK+09, KTK14, and TK11 waveforms, respectively. Note in these figures that the distance to the source $(d)$ is set differently as $d=2 \mathrm{kpc}, 10 \mathrm{kpc}$, and $50 \mathrm{kpc}$ each for Figure 6, 7, and 8, respectively, by which the optimal SNR exceeds 8 (e.g., Table If bearing in mind that signal detection should be preconditioned for the localization of the source. 
In the skymaps, the color scale corresponds to the area of the error regions, so that the smaller values (bluish regions) or high values (reddish regions) correspond to good or bad accuracy regarding the skymap reconstruction. Comparing Figure 6 with Figures 7 and 8, one can see from the area of bluish regions that the accuracy is biggest for the KK+09 waveform (Figure 6) at $d=2 \mathrm{kpc}$, which is followed roughly in order by the TK11 at $d=50 \mathrm{kpc}$ (Figure 8) and the KTK14 waveform at $d=10 \mathrm{kpc}$ (Figure 7). The accuracy of the skymap reconstruction depends on many ingredients, such as the signal strength and duration, waveform morphology, etc. For our three sets of the signals, the source localization (positioned at the optimal distance) turns out to be most accurately determined for the KK+09 waveform, which may be rather counter-intuitive due to the absence of the distinct peaks in the waveform. However, this is mainly due to the longer simulation time compared to the other two waveforms. This kind of ambiguity is typical for the least constrained unmodeled search and networks with only four spatially separated detectors (e.g., [131]).

For the $\mathrm{KK}+$ waveforms, the distribution and the area of the error regions are almost similar between the 3D model without or with rotation or either seen from equator or pole. As mentioned, this is due to the assumed initial small rotation rate. The region, which we call as hot spots: the source localization there is not good (colored by red or yellow in the skymap), is confined in small clusters in this case. On the other hand, the hot spots are distributed over a large area and split into more smaller clusters in the KTK14 waveforms (Figure 7).

The TK11 signals (Figure 8) from 2D MHD models were assumed to be positioned at $d=50 \mathrm{kpc}$ and seen from the equator of the source. Comparing the left (model B12X5 301 ) with right panel (model B12X5 $\beta 10$ ), the area of the error regions is shown to be smaller for the model with the larger initial rotation rates (right panel). This feature is clearly seen in the rest of the $2 \mathrm{D}$ models with rapid rotation.

In this study, the number of the examined multi-D models is rather limited (17) and it is truly far from comprehensive. At this stage, we are only able to discuss how well we can localize the GW signals for the limited set of the CCSN models. To seek for some systematic trend, we summarize in Table II the range of the solid angle within which the (given) position reconstruction can be done. It is shown in the table that the accuracy of the sky position reconstruction is generally higher for models with rapid rotation (e.g., model R3 of KTK14 and models from TK11) than for the non-rotating models (e.g., model R0 of 
KTK14 and models from $K K+9)$. Finally we point out that the configuration of the $\mathrm{H}-\mathrm{L}-\mathrm{V}-\mathrm{K}$ network is fine because the sky position of the Galactic center (horizontal yellow line in the skymap) does not always coincide with the hot spots as shown in Figures 6- 8 .

TABLE II. Fraction of the solid angle relative to the whole sky $(4 \pi)$, within which the position reconstruction can be done by each of the angular resolution $(d \Omega=5 \times 5,10 \times 10$ square degrees, and so on).

\begin{tabular}{llllll}
\hline \hline Model & $5 \mathrm{deg}$ & $10 \mathrm{deg}$ & $20 \mathrm{deg}$ & $25 \mathrm{deg}$ & $30 \mathrm{deg}$ \\
\hline KK+9 Ae & 0.2304 & 0.3926 & 0.5832 & 0.6472 & 0.6899 \\
KK+9 Ap & 0.3414 & 0.5249 & 0.7084 & 0.7468 & 0.7952 \\
\hline KTK14 R0e & 0.6558 & 0.8250 & 0.9289 & 0.9488 & 0.9545 \\
KTK14 R0p & 0.5533 & 0.7411 & 0.8734 & 0.9004 & 0.9232 \\
KTK14 R2e & 0.1422 & 0.3272 & 0.5306 & 0.5832 & 0.6430 \\
KTK14 R2p & 0.4893 & 0.7212 & 0.8535 & 0.8962 & 0.9260 \\
KTK14 R3e & 0.6714 & 0.8492 & 0.9644 & 0.9844 & 0.9957 \\
KTK14 R3p & 0.6330 & 0.8805 & 0.9758 & 0.9872 & 0.9986 \\
\hline TK11 B12X1 $\beta 01$ & 0.5007 & 0.7112 & 0.8321 & 0.8606 & 0.8834 \\
TK11 B12X1 $\beta 10$ & 0.4979 & 0.7084 & 0.8450 & 0.8691 & 0.8890 \\
\hline \hline
\end{tabular}

\section{Waveform reconstruction}

Figures 9 and 10 show comparison between the reconstructed (red lines) and the original waveforms of the two representative models with rapid rotation (black lines, for the + mode (upper) and for the $\times$ mode (lower), respectively). Seen from the pole of model R3 (i.e., model R3p) in the KTK14 catalogue (top two pair panels of Figure 9), the matched-filtering SNR of the reconstructed $h_{+}, h_{\times}$reach to $\sim 30$ and $60(d=10 \mathrm{kpc})$, respectively. Seen from the equator (bottom two pair panels of Figure 9), the SNR of the (conventional) bounce GW signal from rapidly rotating collapse and bounce $\left(h_{+}\right.$, upper part of the bottom two panels) reaches $\sim 35-38$. Remembering that any coherent network analysis has bias and one cannot completely correct the bias in general, this result is not as bad compared with the ideal SNRs of $\sim 60$ for an optimally oriented and optimally located source (Table II). 

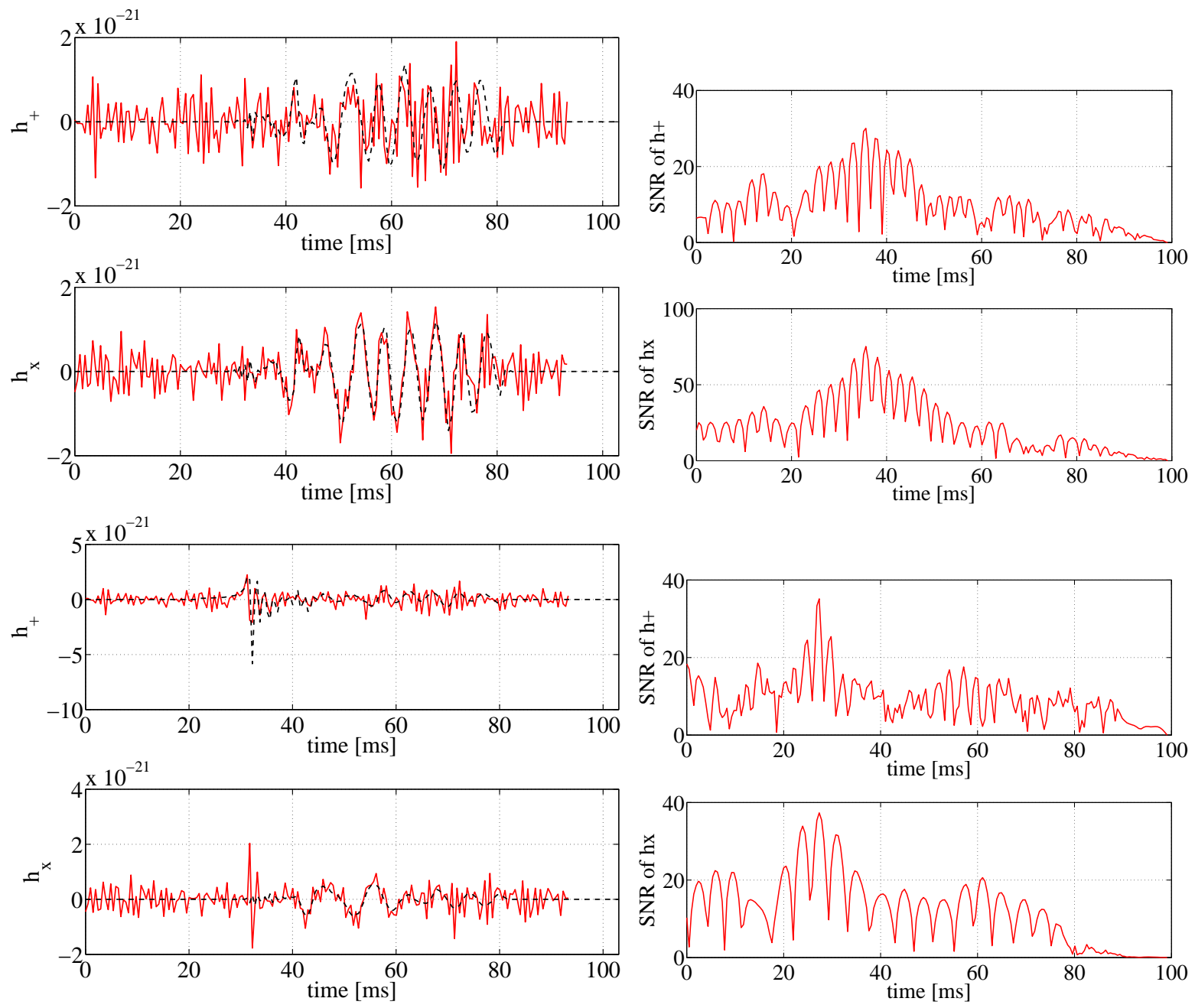

FIG. 9. Left panels show the reconstructed $h_{+}$(upper and $h_{\times}$(lower) waveforms (red lines) of model R3p (top two panels) and R3e (bottom two panels) $(d=10 \mathrm{kpc})$. The black line corresponds to the injected (original) waveform. Right panels show the corresponding output of matched filtering for the reconstructed signals (compare with the left panels), in which the output is the SNR.

From Figure 10, one can also see that the bounce signal is well reconstructed by the RIDGE pipeline (with the matched-filtering SNR reaching to 40 (bottom panel) for the 2D MHD model from the TK11 catalogue. It is also shown that the quasi-monotonically increasing component in the original waveform (black line after $\sim 20 \mathrm{~ms}$ postbounce) disappears in the reconstructed waveform (red line, top panel). This again reflects that such low-frequency component is hard to detect due to seismic noises. Regarding a 3D (non-rotating) model (not shown) from the $\mathrm{KK}+$ catalogue, the detection efficiency of the high-frequency component 

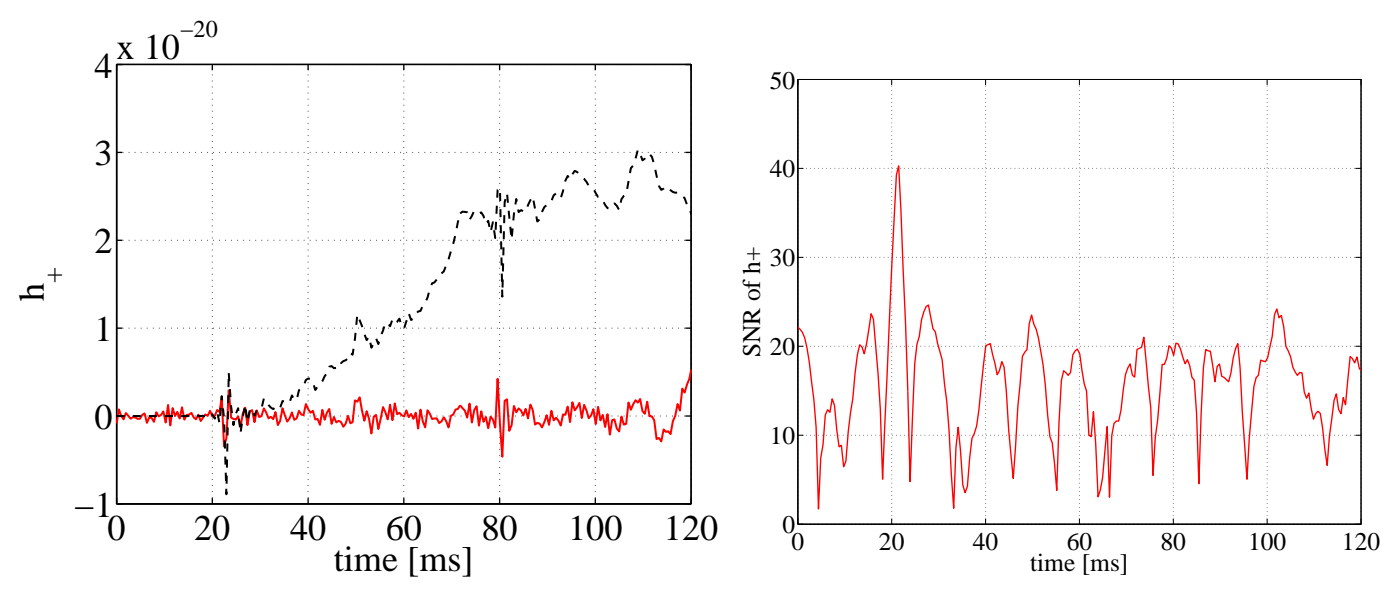

FIG. 10. Same as Figure 9 but for model B12X1ß10 from the TK11 catalogue.

(with the variation timescale of $\mathrm{ms}$ ) is not high compared to those for Figures 9 and 10 because of the absence of the distinct waveform morphology. On the other hand, the shape of the waveform with the variation timescale of 50 - $100 \mathrm{~ms}$ (which closely corresponds to the SASI modulation) is captured to some extent, by which the matched-filtering SNR is in the range from $\sim 5$ to 10 for the nearby source $(d=2 \mathrm{kpc}$ in this case).

\section{INFERRING THE POSTBOUNCE HYDRODYNAMIC EPISODES}

By performing the GW spectrogram analysis (e.g., [71, 76, 81]), we move on to discuss what information we could extract about the hydrodynamic episode in the postbounce core.

For the sake of simplicity, we first focus on the waveform of a 2D MHD model from the TK11 catalogue. The top left panel of Figure 11 is the spectrogram of the original waveform. Colored by red, there are three distinct excess at the simulation time $t_{\text {sim }} \sim 20 \mathrm{~ms}, 60 \mathrm{~ms}$, and $90 \mathrm{~ms}$, respectively. The first excess peaking around $1 \mathrm{kHz}\left(t_{\mathrm{sim}} \sim 20 \mathrm{~ms}\right)$ comes from the bounce signal, which is followed by the excess at $t_{\text {sim }} \sim 60 \mathrm{~ms}$ (we call this as the second excess) due to a relatively large ring-down. The third excess $\left(t_{\text {sim }} \sim 90 \mathrm{~ms}\right)$ which extends to a lower frequency regime $(\lesssim 100 \mathrm{~Hz})$ comes from the second core bounce (e.g., Figure 10 ) and the subsequent formation of the secondary MHD jet (see, [54] for more details).

Here it should be noted that these hydrodynamic features imprinted in the spectrogram can be seen also in the spectrogram for the reconstructed signals (top right panel of Figure 11). Comparing the top left with the top right panel, the high frequency domain (colored by 

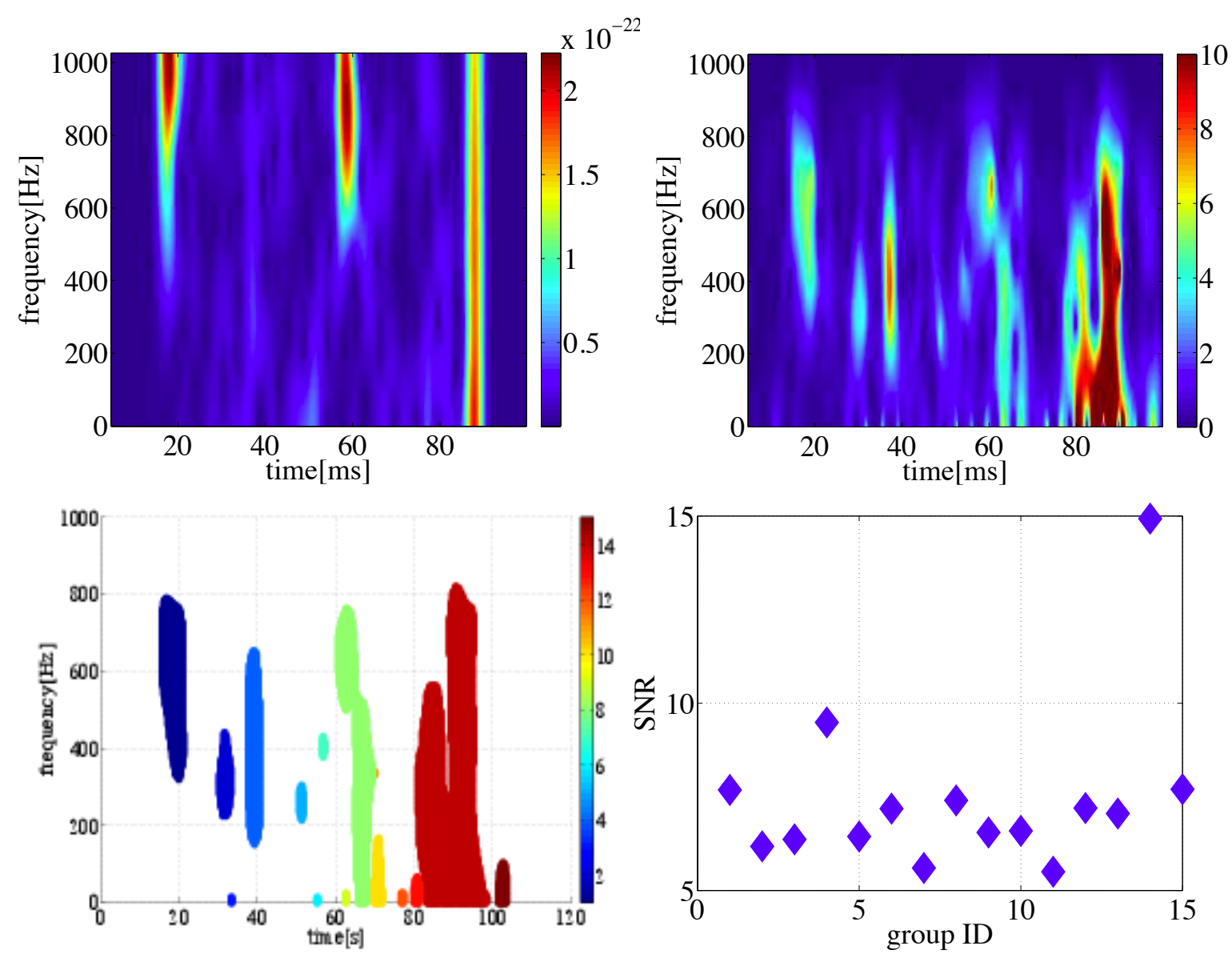

FIG. 11. Top left panel is the GW spectrogram of the injected signal (theoretical prediction) showing the amplitude at $d=10 \mathrm{kpc}$ as a function of simulation time (horizontal axis) and frequency (vertical axis) for model TK11B12X1ß01 from the TK11 catalogue. Same as the top left panel, but the top right panel is the spectrogram of the reconstructed waveform, in which the color scale represents the time-frequency $\operatorname{SNR}\left(\mathrm{SNR}_{\mathrm{TF}}\right.$, see text for the definition). The bottom left panel shows the groups of the excess with the same $\mathrm{SNR}_{\mathrm{TF}}$ with the color scale representing the grouping identification (ID) number. The bottom right panel shows the grouping SNR (see text) as a function of the group identification (ID) number.

red in the top left panel from $\sim 800$ to $1 \mathrm{kHz}$ in each of the above three excesses) disappear in the top right panel. This is because these frequency domain is out of the highest detectors' sensitivity that is limited by quantum noises (e.g., Figure 2). In the top right panel, the color scale represents the ratio of the GW amplitude with both the model prediction and the detector's noise to that with the noise only (without the model prediction). We call this 
quantity as the time-frequency $S N R\left(\mathrm{SNR}_{\mathrm{TF}}\right)$ because it is defined in each pixel of the timefrequency $(d t-d f)$ domain representing the strength of the signal relative to the noise. The pixel resolution taken here is $20 \mathrm{~ms}$. In this analysis, each time-frequency tile is overlapped except for 1 pixel, the group SNR of each time-frequency tile is averaged over the overlapped tiles.

By selecting the pixels with the same time-frequency SNR (exceeding 5), we can divide the patchy excesses in the spectrogram into several disconnected groups (bottom left panel of Figure 11). The color scale of the bottom left panel represents the identification (ID) number of each of the groups (which we call as the grouping ID). By summing up the time-frequency SNR in the disconnected area $(S)$ (having the same $\mathrm{SNR}_{\mathrm{TF}}$ )), we define the grouping $S N R$ (i.e., $\mathrm{SNR}_{\text {group }} \equiv \int_{S} d t d f \mathrm{SNR}_{\mathrm{TF}}$ ). The bottom right panel of Figure 11 shows the $\mathrm{SNR}_{\text {group }}$ as a function of the grouping ID. It should be noted that the identification of distinct clusters in the GW spectrograms presented in this work is nothing but a very rough and optimistic estimate because we consider only the idealized Gaussian noise. Effects of realistic noise need to be considered, which is one of the most important tasks to be studied as a sequel of this work.

The first excess near at bounce (shown as a blue prolate region at $t_{\text {sim }} \sim 20 \mathrm{~ms}$, bottom left panel) has the group ID = 1 (blue in the color-scale), which is shown to have the grouping SNR 7.5 (e.g., bottom right panel). The second and third excess $\left(t_{\text {sim }} \sim 60,90 \mathrm{~ms}\right)$ has the group ID $=4$ and 14 , the $\mathrm{SNR}_{\text {group }}$ of which is 9.6 and 15 , respectively. When we set the detection threshold as 8 , the bounce signature is expected to be detectable to $9.4 \mathrm{kpc}$ for the H-L-V-K observation, while the (strong) ring-down of the PNS (the second excess) and the subsequent bounce and the formation of the recurrent MHD jets (the third excess) can be detected to $12 \mathrm{kpc}$ and $19 \mathrm{kpc}$, respectively.

As one would imagine, the spectrogram of the $\mathrm{KK}+$ waveforms, as contrary to the TK11/KTK14 waveforms, does not possess clear excess. Since it turns out to be difficult to perform the grouping analysis as in Figures 11, we limit ourselves to focus on the KTK14 waveforms in the following.

Figures 12 and 13 show the similar analysis for the waveforms of model R3, the most rapidly rotating model in the KTK14 catalogue, either seen from equator (R3e) or pole (R3p), respectively.

For the equatorial observer (Figure 12), a clear excess in the spectrogram is seen in the + 
mode (top left panel) at $t_{\text {sim }} \sim 35 \mathrm{~ms}$ (red prolate region), which corresponds to the epoch of rotating core bounce. The quasi-oscillatory period of the waveform near bounce is $1 \sim 5$ ms (e.g., middle panel of Figure 1), which accounts for the excess in the frequency range of $200-1000 \mathrm{~Hz}$ in the spectrogram (top left panel).

This (best-studied) rotating bounce signal is hardly seen in the $\times$ mode for the equatorial observer (top right panel) nor for the polar observer (top panels of Figure 13) with both polarizations $(+($ left $)$ or $\times($ right $)$ mode, respectively). Clearly seen for the polar observer is an another excess that appears in the spectrograms between $t_{\text {sim }}=40 \sim 80 \mathrm{~ms}$ peaking around the frequency of $200 \mathrm{~Hz}$ (top panels in Figure 13). As elaborately discussed in [80, this characteristic frequency $(\sim 200 \mathrm{~Hz})$ comes from the growth of one-armed spiral instabilities in the vicinity of the rapidly rotating PNS (see also the periodic waveform patterns in the middle panel of Figure 1).

In the reconstructed waveforms (second columns of Figures 12 and 13), it can be seen that the GW signatures seen in the injected signals due to rotating bounce (top left panel in Figure 12) and the spiral instabilities (top panels in Figure 13) are still present, although the excess in the spectrograms becomes especially weak for the bounce signal at the high frequency regime (compare the left panels in the first and second columns of Figure 12).

Regarding the + mode seen from the equator (left panels of Figure 12), $\mathrm{SNR}_{\mathrm{TF}}$ of the rotating bounce signal is 14.3 (second panel (left)), which is assigned to have the ID number 1 (red prolate region in the third column (left)). Setting again the detection threshold as 8 with respect to $\mathrm{SNR}_{\text {group }}$, the detection horizon extends to $17.9 \mathrm{kpc}$. Regarding the GW signature from the spiral instabilities, the corresponding excess $\left(\mathrm{SNR}_{\mathrm{TF}}=13.8\right)$ is assigned to have the ID number 2 and 3 (yellow/orange region in the third column (left)). The horizon distance is $\sim 17.3 \mathrm{kpc}$. As for the $\times$ mode (right panels of Figure 12), SNR group $_{\text {is }}$ 9.0 (the ID number is 1) and 10.3 (the ID number is 2, which has biggest SNR among the candidate IDs of 2,3 , and 4 (third column (right)), the detection horizon of which is 11.3 $\mathrm{kpc}$ and $12.9 \mathrm{kpc}$, respectively.

Seen from pole (Figures 13), the excess in the spectrogram of the model R3 waveform (top panels) is categorized into three $\left(\mathrm{SNR}_{\mathrm{TF}}=29.3,31.7\right.$, and 26.7 , third column (left, + mode $)$ ) or two $\left(\mathrm{SNR}_{\mathrm{TF}}=16.1\right.$ and 15.2 (third column (right, $\times$ mode $)$ ). Choosing the biggest $\mathrm{SNR}_{\mathrm{TF}}$ among the groups, the detection horizon of the + and $\times$ mode turns out to extend up to $\sim 40$ and $20 \mathrm{kpc}$, respectively. 

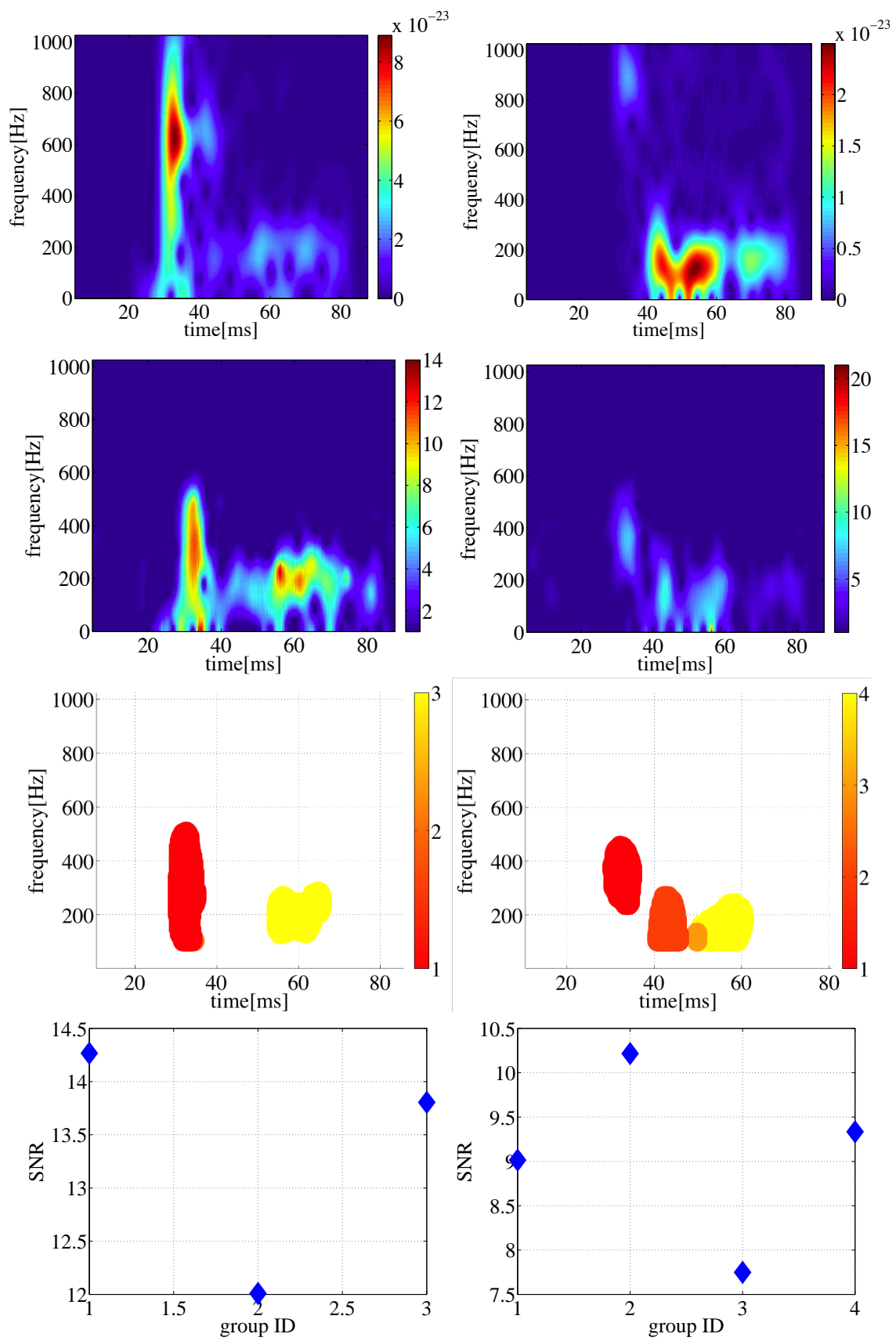

FIG. 12. Similar to Figure 11 but for model R3 seen from equator from the KTK14 catalogue. The left and right panels correspond to the waveform with + and $\times$ mode, respectively. The first and second column corresponds to the spectrograms of the injected and reconstructed signals, respectively. The third and fourth column show the spectrogram of $\mathrm{SNR}_{\mathrm{TF}}$ and the $\mathrm{SNR}_{\text {group }}$ with the ID number, respectively. 
Finally, Table III summarizes the horizon distances of all the rotating models in the KTK14 catalogue. Regarding the rotating bounce signals (labelled as "Rotating core-bounce" in the table), the horizon distance becomes longest $(\sim 18 \mathrm{kpc})$ seen from equator for the most rapidly rotating model (R3) by the $\mathrm{H}-\mathrm{L}-\mathrm{V}-\mathrm{K}$ network considered in this work. In order that the SNR exceeds 8 (to claim detection), the initial rotation rate should be higher than $\Omega_{\text {ini }}=\pi / 2(\mathrm{rad} / \mathrm{s})$ (model R2) among the 3D-GR models. Regarding the GW signals from non-axisymmetric instabilities, the horizon distances become generally longer when seen from pole than seen from equator. This is because the low-mode instabilities characterized by the spiral arms develop most preferentially in the equatorial plane. The maximum horizon distance extends up to $\sim 40 \mathrm{kpc}$ for the most rapidly rotating model (R3). The horizon distance does not decrease monotonically with the initial rotation rate. In fact, comparing the initial rotation rate of model $\mathrm{R} 3\left(\Omega_{\mathrm{ini}}=\pi(\mathrm{rad} / \mathrm{s})\right)$ and that of model $\mathrm{R} 2$, the maximum horizon distance of model R2 $(\sim 36 \mathrm{kpc})$ is relatively close to that of model R3 ( $\sim 40 \mathrm{kpc})$.

From Table III, we speculate that the chance of detecting GWs from rapidly rotating CCSNe could become quite higher for the quasi-periodic signals inherent to the nonaxisymmetric instabilities than for the short-duration signals emitted at rotating collapse and bounce. As repeatedly mentioned before, it should be cautioned again that the numbers in Table III are based on a very optimistic estimate using the idealized Gaussian detector noise, and they should be interpreted as an upper bound of the horizon distance. For a more quantitative investigation, one also needs a more accurate waveform prediction based on long-term 3D-GR models with sophisticated neutrino transport, toward which we have attempted to make the very first step in this study.

\section{SUMMARY AND DISCUSSIONS}

Using predictions from 3D hydrodynamics simulations of CCSNe, we presented a coherent network analysis to detection, reconstruction, and the source localization of the GW signals. The network considered in this work consisted of the LIGO Hanford, LIGO Livingston, VIRGO, and KAGRA interferometers. We first computed the SNR and the optimistic detectability of the GW signals with a very idealized situation (i.e., only a single detector for

an optimally oriented and optimally located source). Then we considered a more realistic situation using the RIDGE coherent network analysis pipeline, in which the multiple detec- 
TABLE III. Model summary of optimistic horizon distances based on the spectrogram analysis. In the first column, GW emission for models from the KTK14 catalogue is categorized either due to rotating core bounce (emitted within $10 \mathrm{~ms}$ postbounce) and due to the subsequent growth of the non-axisymmetric instabilities in the vicinity of the PNS. By setting the detection threshold as $\mathrm{SNR}_{\text {group }}=8$, the horizon distance is given for each of the 3D-GR models for the equatorial or polar observer (labelled such as by R3e and R3p) with + or $\times$ polarization. The blank "_." represents that the $\mathrm{SNR}_{\text {group }}$ does not exceed the threshold.

\begin{tabular}{lcc}
\hline \hline Model & Rotating core-bounce & Non-axisymmetric instabilities \\
\hline R3e & $17.9 \mathrm{kpc}(+), 11.3 \mathrm{kpc}(\times)$ & $17.3 \mathrm{kpc}(+), 12.9 \mathrm{kpc}(\times)$ \\
R3p & - & $39.4 \mathrm{kpc}(+), 20.1 \mathrm{kpc}(\times)$ \\
R2e & $14.0 \mathrm{kpc}(+)$ & $16.5 \mathrm{kpc}(+)$ \\
R2p & - & $35.9 \mathrm{kpc}(+), 14.0 \mathrm{kpc}(\times)$ \\
R1e & - & $16.8 \mathrm{kpc}(+), 7.6 \mathrm{kpc}(\times)$ \\
R1p & - & $5.9 \mathrm{kpc}(+), 11.1 \mathrm{kpc}(\times)$ \\
\hline \hline
\end{tabular}

tors were used for an arbitrary oriented source. By combining with the GW spectrogram analysis, it was shown that several important hydrodynamics features imprinted in the original waveforms persist in the waveforms of the reconstructed signals. The characteristic excess in the GW spectrograms originates not only from rotating core-collapse and bounce, the subsequent ring down of the PNS as previously identified, but also from the formation of MHD jets and non-axisymmetric instabilities in the vicinity of the PNS. Regarding the GW signals emitted near at the rotating core bounce, the horizon distance, which we optimistically set by a SNR exceeding 8, extends up to $\sim 18 \mathrm{kpc}$ for the most rapidly rotating 3D model among the employed waveform libraries. Only for models with the precollapse angular velocity higher than $\Omega_{0}=\pi / 2(\mathrm{rad} / \mathrm{s})$, the SNRs of the rotating bounce signals exceed the fiducial detection threshold. Following the rotating core bounce, the dominant source of the GW emission shifts to the non-axisymmetric instabilities that develop in the region between the stalled shock and the PNS. It was pointed out that the horizon distances from the non-axisymmetric instabilities are generally longer when seen from the direction parallel to the rotational axis of the source than seen from the equator. This is because the spiral arms that are inherent to the low-modes instabilities develop more preferentially in the 

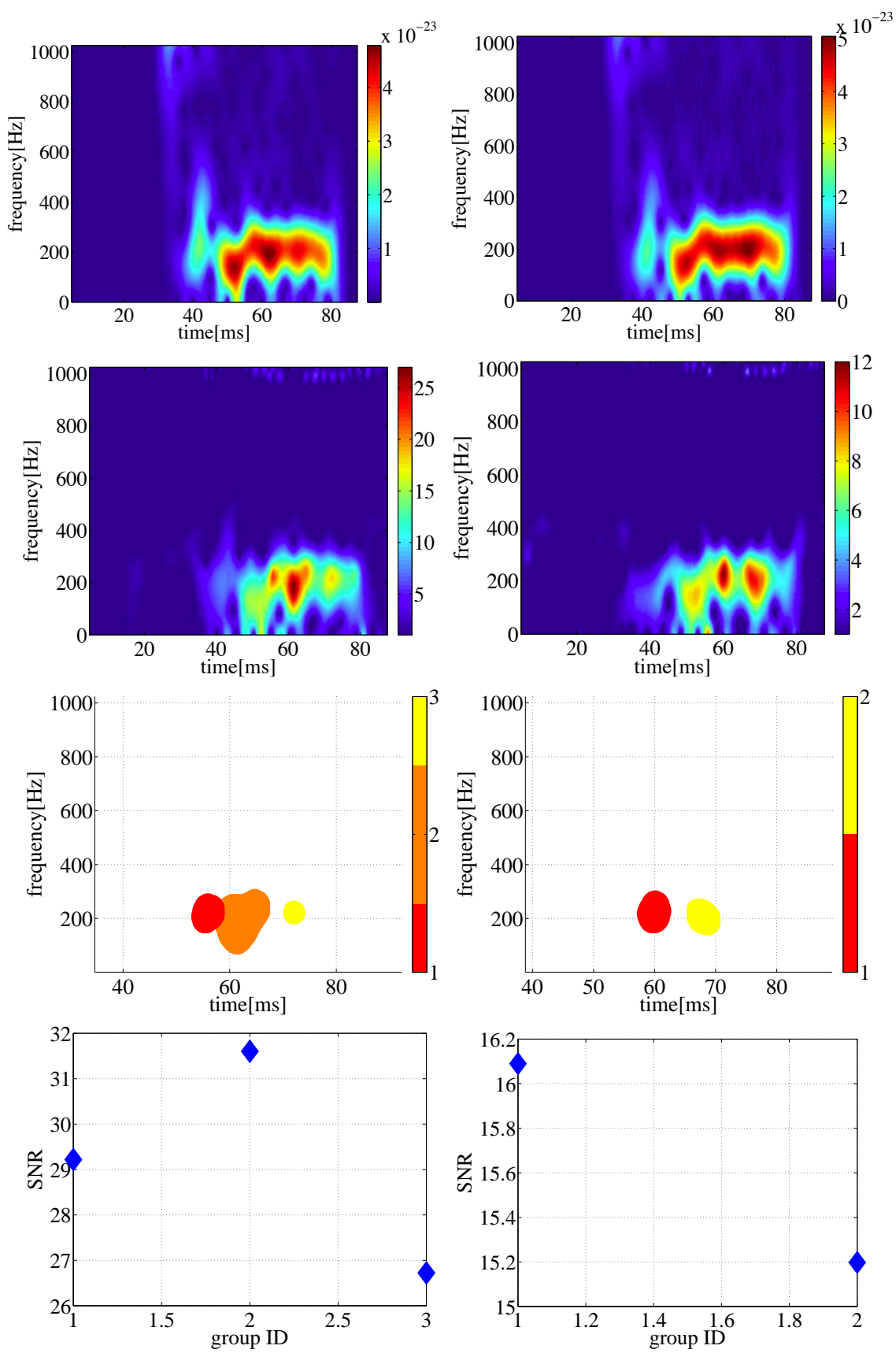

FIG. 13. Same as Figure 12 but for model R3 seen from pole.

equatorial plane. Among the 3D general-relativistic models in which the non-axisymmetric instabilities set in, the horizon distances extend maximally up to $\sim 40 \mathrm{kpc}$ seen from the pole and they are rather insensitive to the imposed initial rotation rates. In addition to the best studied GW signals due to rotating core-collapse and bounce, it was suggested that the quasi-periodic signals due to the non-axisymmetric instabilities and the detectability should 
deserve further investigation to elucidate the inner-working of the rapidly rotating CCSNe.

While we have shown that the spectrogram analysis is effective for the GW signals from rapidly rotating collapse, which is most likely to be associated with the MHD-driven mechanism, the ability on the stochastic waveforms from the neutrino mechanism remains to be tested. Recently it was demonstrated by 2D-GR models with elaborate transport scheme [43. that a violent mass accretion to the PNS leads to an efficient GW emission in the late postbounce phase, which can be nicely explained by the buoyant frequency near the PNS surface [76]. Such a high-frequency feature $(\sim 100$ to $\sim 1 \mathrm{kHz})$ are generic in 2D explosion models, which is expected to be also the case in 3D [81]. With a growing supercomputing power and a rapid development of CCSN modeling (e.g., [? ? ? ? ? ), we speculate that the construction of dense waveform catalogues based on self-consistent 3D-GR models is becoming a reality in the decade to come [34, 35]. We hope that in combination of the refined waveform predictions the GW analysis schemes will be also updated such as by taking a coincidence with neutrino signals ([132, 133], e.g., [134? ] for a review), which should be indispensable to decipher the CCSN mechanism from the multi-messengers observables in the NEXT nearby event.

\section{ACKNOWLEDGMENTS}

$\mathrm{KH}$ would like to thank B. Allen for warm hospitality during his stay in Hannover and S.D. Mohanty for valuable comments and encouragement. We are grateful to N. Kanda and the members in his lab in Osaka-city university for helpful discussions. KK and TT are thankful to K. Sato and S. Yamada for continuing encouragements. Numerical computations were carried out in part on XC30 and general common use computer system at the center for Computational Astrophysics, CfCA, the National Astronomical Observatory of Japan, Oakleaf FX10 at Supercomputing Division in University of Tokyo, and on SR16000 at YITP in Kyoto University. This study was supported in part by the Grants-in-Aid for the Scientific Research from the Ministry of Education, Science and Culture of Japan (Nos. 24103006, 24244036, 26707013, and 26870823) and by HPCI Strategic Program of Japanese MEXT. 


\section{APPENDIX A, WAVEFORM CATALOGUES}

\section{The $\mathrm{KK}+$ waveforms}

The top panels of Figure 1 show an example waveform (left panel) and a snapshot (right panel) of a 3D model that is trending towards an explosion by the neutrino mechanism [74, 75, 100]. In the 3D model, the neutrino luminosity $\left(L_{\nu}\right)$ from the PNS was treated as a parameter to trigger explosions and the initial conditions were derived from a steady-state approximation of the postshock structure and the Newtonian hydrodynamics outside an inner boundary at $50 \mathrm{~km}$ was solved. The core neutrino luminosity is taken as $L_{\nu}=6.8 \times 10^{52}$ and $6.4 \times 10^{52} \mathrm{erg} / \mathrm{s}$ for the $\mathrm{KK}+09$ (corresponding to model A in [74]) and the $\mathrm{KK}+11$ waveform (model C2 in [100], top panels in Figure 1), respectively. In model KK+11, stellar rotation was taken into account by adding a uniform rotation to the flow at the outer boundary of the computational domain (e.g., [101]), the angular momentum of which is assumed to agree with recent stellar evolution models [102].

The top right panel is a snapshot (at $t_{\text {sim }}=513 \mathrm{~ms}$ ) seen from the rotational axis for model C2. Note that the time $\left(t_{\text {sim }}\right)$ is measured from the epoch when simulations are started. The first and third quadrants of the panel show the profiles of the high-entropy bubbles (colored by red) inside the surface of the standing shock wave (the second and fourth quadrants). The side length of the panel is $1000 \mathrm{~km}$. The high-entropy bubbles are seen to develop like a spiral arm, which is a signature of the spiral SASI modes. Under the influence of the spiral and sloshing SASI modes and neutrino-driven convection, the 3D model starts to be exploding at $t_{\text {sim }} \sim 200 \mathrm{~ms}$ after the stalled shock comes to a steady state. In the top left panel, the waveforms only $t_{\text {sim }} \gtrsim 200 \mathrm{~ms}$ is shown because the amplitudes are zero (or very small) before the non-spherical hydrodynamical instabilities enter to the non-linear phase (see also the inset of the top right panel). As shown, the wave amplitudes change stochastically with time, because the non-sphericities in the postshock region are essentially governed by turbulent flows. Note that the wave amplitudes of our simplistic 3D models are qualitatively in agreement with those obtained in more realistic 3D models [103.

The inset of the top right panel shows the waveform contributed only from anisotropic neutrino emission (pink line (seen from pole, + mode), green line (seen from pole, $\times$ mode), light blue line (seen from equator, + mode), and red line (seen from equator, $\times$ mode, 
respectively). As already discussed in [19, 74, 75, 100, 104, 105], the time variability of the neutrino(-originated) GWs is much longer ( $\gtrsim O(10) \mathrm{ms})$ due to the memory effect [106] than that of the matter GWs $(\lesssim O(10)$ ms (top left panel)). As a result, the peak frequencies of the neutrino GWs are typically below $\sim 100 \mathrm{~Hz}$. These low frequency GWs are very difficult to detect by ground-based detectors whose sensitivity is limited mainly by seismic noises. In the case of KAGRA, the sensitivity at $20 \mathrm{~Hz}$ is $5 \times 10^{-23}$, which is about 20-times worse than the most sensitive frequency domain around $80-200 \mathrm{~Hz}$. In the following, we thus focus on the matter GW signals that are more important in discussing the detectability.

\section{The KTK14 waveforms}

The middle panels of Figure 1 show the waveform (left panels) and the 3D entropy plot (49 ms postbounce) of a rapidly rotating model (R3) in Kuroda et al. [80]. For this model, an angular velocity of $\Omega_{0}=\pi \mathrm{rad} / \mathrm{s}$ is added to the non-rotating $15 M_{\odot}$ progenitor of [107] with a quadratic cut-off parameter at the radius of $X_{0}=1000 \mathrm{~km}$. Shortly after bounce $(\sim 15$ ms postbounce), one-armed spiral modes were observed to develop in the postshock region for this rapidly rotating model. As a result, the waveforms show narrow-band and highly quasi-periodic signals (regardless of the GW polarizations, see the left middle panels), which persist until the end of simulations $\left(t_{\mathrm{sim}} \sim 60 \mathrm{~ms}\right)$. Since the typical frequency of the quasi-

periodic waveform can be well explained by the propagating acoustic waves between the stalled shock and the rotating PNS surface, the waveforms are most likely to be associated with the appearance of the spiral SASI (see Kuroda et al. [80] for more detail). Regarding the + mode of the signal seen from equator (not shown in Figure 1), typical GW features of the so-called type I waveforms (e.g., [108]) were clearly seen [80], i.e., a first positive peak just before bounce precedes the deep negative signal at bounce, which is followed by the subsequent ring-down phase.

In addition to the rapidly rotating model (R3), we use three waveforms of more slowly rotating models from [80], which correspond to models $\mathrm{R} 0\left(\Omega_{0}=0 \mathrm{rad} / \mathrm{s}\right.$ (non-rotating)), R1 $\left(\Omega_{0}=\pi / 6 \mathrm{rad} / \mathrm{s}\right)$, and $\mathrm{R} 2\left(\Omega_{0}=\pi / 2 \mathrm{rad} / \mathrm{s}\right)$, respectively. All of the 3D models are based on full GR hydrodynamic simulations, in which an approximate three-flavour neutrino transport was solved with the use of an analytical closure scheme (e.g., [109]). The wave amplitudes for the non-rotating (model R0) and slow-rotating (model R1) stay much smaller $\left(\lesssim 3 \times 10^{-22}\right)$ 
during the simulation time ( $\lesssim 50 \mathrm{~ms}$ postbounce). These GW amplitudes and frequencies are consistent with 3D (post-)Newtonian [79] or GR models [66, 110] with more idealized transport scheme and 2D GR models with more detailed neutrino transport [82].

\section{The TK11 waveforms}

To discuss GWs from models that produce MHD explosions, we use four waveforms from [54]. The authors performed 2D special-relativistic (SR) MHD simulations with the use of an approximate GR potential [111, in which a neutrino leakage scheme was employed to take into account neutrino cooling [112]. The computed models were named with the first part, B12, representing the strength of the initial magnetic field parallel to the spin axis $\left(10^{12} \mathrm{G}\right)$, the second part, X1, X5, or X20, indicating the degree of differential rotation $\left(X_{0}\right.$ $=100,500,2000 \mathrm{~km}$, respectively), and the third part, $\beta=0.1$ or 1 , showing the rotation parameter (the ratio of the rotational energy to the absolute value of the gravitational energy prior to core collapse).

The bottom left panel of Figure 1 shows that the waveform from MHD explosions tends to have a quasi-monotonically increasing component, which is followed by the typical type I GW signature near at bounce $\left(0.02 \lesssim t_{\text {sim }} \lesssim 0.03 \mathrm{~s}\right)$. Such feature was only observed for models with strong precollapse magnetic field and with rapid rotation initially imposed (e.g., model B12X1 30.1 ). The increasing trend comes from bipolar flows (bottom right panel of Figure 1) as shown by [113, 114]. Again the low frequency waveform $(\lesssim 100 \mathrm{~Hz})$ are hard to detect, but it may be worth mentioning here that future space interferometers like FabryPerot type DECIGO are designed to be sensitive in the frequency regimes [115, 116].

The GW amplitudes and frequencies of the TK11 catalogue are consistent with those obtained in previous 2D [113, 114] and 3D [79] MHD models. We chose to take the signal predictions from 2D models in order to compare the wave amplitudes with those in 3D models (i.e., KTK14 catalogue), and also to discuss how difficult it is to detect the low frequency components for ground-based interferometers even by performing the coherent network analysis. 


\section{APPENDIX B, COHERENT NETWORK ANALYSIS}

In order to compute the signal detection, reconstruction, and source localization of the model waveforms in the last section, we perform a coherent network analysis using a pipeline called RIDGE (see [94] for details). In the algorithm, one combines information from multiple GW detectors coherently to perform a maximum likelihood analysis, taking into account the antenna patterns, geographical locations of the detectors, and the sky direction to the source ([93, 94, 120-124 and therein). The RIDGE pipeline takes full advantage of the global network of currently working and future interferometers (LIGO Hanford (H), LIGO Livingston (V), VIRGO (V), and KAGRA (K)), resulting in enhanced detection efficiency. For the detailed description of the pipeline, see Refs. [94, 125].

Using the RIDGE pipeline, we perform Monte Carlo simulations (see [94, 125] for more details) to investigate the detectability of the model waveforms in section $\mathrm{V}$. For the detector noise spectrum densities of the four detectors $(\mathrm{H}, \mathrm{L}),(\mathrm{V})$, and $(\mathrm{K})$, we use the ones in [8, 129, 130] and keep the locations and orientations the same as the real detectors. Gaussian, stationary noise was generated by first generating four independent realizations of white noise with the sampling frequency of $2048 \mathrm{~Hz}$ and then passing them through the FIR filters having transfer functions that approximately match the design curves.

Signals placed at a distance of $10 \mathrm{kpc}$ from the earth were added to the simulated noise at regular intervals. The sky locations where signals are injected in are (longitude,latitude) is $\left(-180^{\circ},-90^{\circ}\right)$ to $\left(180^{\circ}, 90^{\circ}\right)$ with resolution of $\left(10^{\circ}, 10^{\circ}\right)$. The time window for the data analysis is $100 \mathrm{~ms}, 300 \mathrm{~ms}, 1 \mathrm{~s}$, for waveforms in the KTK14, TK11, and KK+09, KK+11 catalogues, respectively. These time windows are much larger than the signal durations, so the detection performance is not optimized. It is possible to obtain higher detection efficiency by optimizing search algorithm, but the optimization is beyond the scope of this paper. The value of the likelihood of the multiple detector data is calculated by changing over the possible sky locations $\hat{\Omega}=(\theta, \phi)$, and the maximum of the likelihoods is chosen. If the maximum likelihood value is above a given threshold, the chosen event candidate is recorded in a detection list. Since the likelihood values are obtained as a function of $\theta$ and $\phi$, this 
two-dimensional output, $\boldsymbol{S}(\theta, \phi)$, is called skymap.

[1] B. P. Abbott, R. Abbott, F. Acernese, R. Adhikari, P. Ajith, B. Allen, G. Allen, M. Alshourbagy, R. S. Amin, S. B. Anderson, and et al., Nature 460, 990 (2009), arXiv:0910.5772 [astro-ph.CO].

[2] J. Abadie et al., Phys. Rev. D 85, 102001 (2010).

[3] B. P. Abbott et al., Astrophys. J. 713, 671 (2010).

[4] J. Abadie, B. P. Abbott, R. Abbott, T. D. Abbott, M. Abernathy, T. Accadia, F. Acernese, C. Adams, R. Adhikari, C. Affeldt, and et al., Phys. Rev. D 85, 082002 (2012), arXiv:1111.7314 [gr-qc].

[5] J. Aasi, J. Abadie, B. P. Abbott, R. Abbott, T. D. Abbott, M. Abernathy, T. Accadia, F. Acernese, C. Adams, T. Adams, and et al., Phys. Rev. D 87, 022002 (2013), arXiv:1209.6533 $[\mathrm{gr}-\mathrm{qc}]$.

[6] J. Aasi, J. Abadie, B. P. Abbott, R. Abbott, T. Abbott, M. R. Abernathy, T. Accadia, F. Acernese, C. Adams, T. Adams, and et al., Phys. Rev. D 88, 122004 (2013), arXiv:1309.6160 [astro-ph.HE].

[7] G. M. Harry and the LIGO Scientific Collaboration, Classical and Quantum Gravity 27, $084006(2010)$.

[8] S. Hild, A. Freise, M. Mantovani, S. Chelkowski, J. Degallaix, and R. Schilling, Classical and Quantum Gravity 26, 025005 (2009), arXiv:0807.2045 [gr-qc],

[9] Y. Aso, Y. Michimura, K. Somiya, M. Ando, O. Miyakawa, T. Sekiguchi, D. Tatsumi, and H. Yamamoto, Phys. Rev. D 88, 043007 (2013), arXiv:1306.6747 [gr-qc].

[10] K. Somiya, Classical and Quantum Gravity 29, 124007 (2012), arXiv:1111.7185 [gr-qc].

[11] M. Punturo, M. Abernathy, F. Acernese, B. Allen, N. Andersson, K. Arun, F. Barone, B. Barr, M. Barsuglia, M. Beker, N. Beveridge, S. Birindelli, S. Bose, L. Bosi, S. Braccini, C. Bradaschia, T. Bulik, E. Calloni, G. Cella, E. Chassande Mottin, S. Chelkowski, A. Chincarini, J. Clark, E. Coccia, C. Colacino, J. Colas, A. Cumming, L. Cunningham, E. Cuoco, S. Danilishin, K. Danzmann, G. De Luca, R. De Salvo, T. Dent, R. De Rosa, L. Di Fiore, A. Di Virgilio, M. Doets, V. Fafone, P. Falferi, R. Flaminio, J. Franc, F. Frasconi, A. Freise, P. Fulda, J. Gair, G. Gemme, A. Gennai, A. Giazotto, K. Glampedakis, M. Granata, 
H. Grote, G. Guidi, G. Hammond, M. Hannam, J. Harms, D. Heinert, M. Hendry, I. Heng, E. Hennes, S. Hild, J. Hough, S. Husa, S. Huttner, G. Jones, F. Khalili, K. Kokeyama, K. Kokkotas, B. Krishnan, M. Lorenzini, H. Lück, E. Majorana, I. Mandel, V. Mandic, I. Martin, C. Michel, Y. Minenkov, N. Morgado, S. Mosca, B. Mours, H. Müller-Ebhardt, P. Murray, R. Nawrodt, J. Nelson, R. Oshaughnessy, C. D. Ott, C. Palomba, A. Paoli, G. Parguez, A. Pasqualetti, R. Passaquieti, D. Passuello, L. Pinard, R. Poggiani, P. Popolizio, M. Prato, P. Puppo, D. Rabeling, P. Rapagnani, J. Read, T. Regimbau, H. Rehbein, S. Reid, L. Rezzolla, F. Ricci, F. Richard, A. Rocchi, S. Rowan, A. Rüdiger, B. Sassolas, B. Sathyaprakash, R. Schnabel, C. Schwarz, P. Seidel, A. Sintes, K. Somiya, F. Speirits, K. Strain, S. Strigin, P. Sutton, S. Tarabrin, A. Thüring, J. van den Brand, C. van Leewen, M. van Veggel, C. van den Broeck, A. Vecchio, J. Veitch, F. Vetrano, A. Vicere, S. Vyatchanin, B. Willke, G. Woan, P. Wolfango, and K. Yamamoto, Classical and Quantum Gravity 27, 194002 (2010).

[12] S. Fairhurst, ArXiv e-prints (2012), arXiv:1205.6611 [gr-qc]

[13] B. S. Sathyaprakash and B. F. Schutz, Living Reviews in Relativity 12, 2 (2009), arXiv:0903.0338 [gr-qc].

[14] J. Faber, Classical and Quantum Gravity 26, 114004 (2009).

[15] M. D. Duez, Classical and Quantum Gravity 27, 114002 (2010), arXiv:0912.3529 [astroph.HE].

[16] N. Andersson, Classical and Quantum Gravity 20, 105 (2003), arXiv:astro-ph/0211057.

[17] C. J. Horowitz, ArXiv e-prints (2011), arXiv:1106.1661 [astro-ph.SR].

[18] K. Kotake, K. Sato, and K. Takahashi, Reports of Progress in Physics 69, 971 (2006), arXiv:astro-ph/0509456.

[19] C. D. Ott, Classical and Quantum Gravity 26, 063001 (2009), arXiv:0809.0695.

[20] C. L. Fryer and K. C. B. New, Living Reviews in Relativity 14, 1 (2011).

[21] S. L. Shapiro and S. A. Teukolsky, Black holes, white dwarfs, and neutron stars: The physics of compact objects (Research supported by the National Science Foundation. New York, Wiley-Interscience, 1983, 663 p., 1983).

[22] B. W. Grefenstette, F. A. Harrison, S. E. Boggs, S. P. Reynolds, C. L. Fryer, K. K. Madsen, D. R. Wik, A. Zoglauer, C. I. Ellinger, D. M. Alexander, H. An, D. Barret, F. E. Christensen, W. W. Craig, K. Forster, P. Giommi, C. J. Hailey, A. Hornstrup, V. M. Kaspi, T. Kitaguchi, J. E. Koglin, P. H. Mao, H. Miyasaka, K. Mori, M. Perri, M. J. Pivovaroff, 
S. Puccetti, V. Rana, D. Stern, N. J. Westergaard, and W. W. Zhang, Nature 506, 339 (2014), arXiv:1403.4978 [astro-ph.HE],

[23] L. Wang, D. A. Howell, P. Höflich, and J. C. Wheeler, Astrophys. J. 550, 1030 (2001).

[24] L. Wang, J. C. Wheeler, P. Höflich, A. Khokhlov, D. Baade, D. Branch, P. Challis, A. V. Filippenko, C. Fransson, P. Garnavich, R. P. Kirshner, P. Lundqvist, R. McCray, N. Panagia, C. S. J. Pun, M. M. Phillips, G. Sonneborn, and N. B. Suntzeff, Astrophys. J. 579, 671 $(2002)$.

[25] K. Maeda, K. Kawabata, P. A. Mazzali, M. Tanaka, S. Valenti, K. Nomoto, T. Hattori, J. Deng, E. Pian, S. Taubenberger, M. Iye, T. Matheson, A. V. Filippenko, K. Aoki, G. Kosugi, Y. Ohyama, T. Sasaki, and T. Takata, Science 319, 1220 (2008), arXiv:0801.1100.

[26] M. Tanaka, K. S. Kawabata, K. Maeda, M. Iye, T. Hattori, E. Pian, K. Nomoto, P. A. Mazzali, and N. Tominaga, Astrophys. J. 699, 1119 (2009), arXiv:0906.1062.

[27] K. Hirata, T. Kajita, M. Koshiba, M. Nakahata, and Y. Oyama, Physical Review Letters 58, 1490 (1987).

[28] R. M. Bionta, G. Blewitt, C. B. Bratton, D. Casper, and A. Ciocio, Physical Review Letters 58, 1494 (1987).

[29] K. Sato and H. Suzuki, Physical Review Letters 58, 2722 (1987).

[30] G. G. Raffelt, Nuclear Physics B Proceedings Supplements 110, 254 (2002), arXiv:hep$\mathrm{ph} / 0201099$.

[31] S. P. Mikheev and A. I. Smirnov, Nuovo Cimento C Geophysics Space Physics C 9, 17 (1986).

[32] H. Duan and J. P. Kneller, Journal of Physics G Nuclear Physics 36, 113201 (2009), arXiv:0904.0974 [astro-ph.HE],

[33] H.-T. Janka, Annual Review of Nuclear and Particle Science 62, 407 (2012), arXiv:1206.2503 [astro-ph.SR].

[34] K. Kotake, K. Sumiyoshi, S. Yamada, T. Takiwaki, T. Kuroda, Y. Suwa, and H. Nagakura, ArXiv e-prints (2012), arXiv:1205.6284 [astro-ph.HE].

[35] A. Mezzacappa, S. W. Bruenn, E. J. Lentz, W. R. Hix, O. E. Bronson Messer, J. A. Harris, E. J. Lingerfelt, E. Endeve, K. N. Yakunin, J. M. Blondin, and P. Marronetti, ArXiv e-prints (2014), arXiv:1405.7075 [astro-ph.SR],

[36] H. A. Bethe and J. R. Wilson, ApJ 295, 14 (1985),

[37] H. A. Bethe, Reviews of Modern Physics 62, 801 (1990), 
[38] M. Rampp and H.-T. Janka, Astrophys. J. Lett. 539, L33 (2000).

[39] M. Liebendörfer, A. Mezzacappa, and F. Thielemann, Phys. Rev. D 63, 104003 (2001).

[40] T. A. Thompson, A. Burrows, and P. A. Pinto, Astrophys. J. 592, 434 (2003).

[41] K. Sumiyoshi, S. Yamada, H. Suzuki, H. Shen, S. Chiba, and H. Toki, Astrophys. J. 629, 922 (2005), arXiv:astro-ph/0506620.

[42] A. Marek and H.-T. Janka, Astrophys. J. 694, 664 (2009), arXiv:0708.3372.

[43] B. Müller, H.-T. Janka, and A. Heger, ApJ 761, 72 (2012), arXiv:1205.7078 [astro-ph.SR].

[44] S. W. Bruenn, A. Mezzacappa, W. R. Hix, E. J. Lentz, O. E. Bronson Messer, E. J. Lingerfelt, J. M. Blondin, E. Endeve, P. Marronetti, and K. N. Yakunin, ApJL 767, L6 (2013), arXiv:1212.1747 [astro-ph.SR].

[45] Y. Suwa, K. Kotake, T. Takiwaki, S. C. Whitehouse, M. Liebendörfer, and K. Sato, PASJ 62, L49+ (2010), arXiv:0912.1157 [astro-ph.HE].

[46] T. Takiwaki, K. Kotake, and Y. Suwa, ApJ 749, 98 (2012), arXiv:1108.3989 [astro-ph.HE],

[47] F. Hanke, B. Müller, A. Wongwathanarat, A. Marek, and H.-T. Janka, ApJ 770, 66 (2013), arXiv:1303.6269 [astro-ph.SR].

[48] T. Takiwaki, K. Kotake, and Y. Suwa, ApJ 786, 83 (2014), arXiv:1308.5755 [astro-ph.SR],

[49] G. S. Bisnovatyi-Kogan, I. P. Popov, and A. A. Samokhin, Ap\&SS 41, 287 (1976).

[50] J. M. LeBlanc and J. R. Wilson, ApJ 161, 541 (1970).

[51] K. Kotake, K. Sato, and K. Takahashi, Reports on Progress in Physics 69, 971 (2006), arXiv:astro-ph/0509456.

[52] A. Burrows, L. Dessart, E. Livne, C. D. Ott, and J. Murphy, ApJ 664, 416 (2007), arXiv:astro-ph/0702539.

[53] T. Takiwaki, K. Kotake, and K. Sato, ApJ 691, 1360 (2009), arXiv:0712.1949.

[54] T. Takiwaki and K. Kotake, ApJ 743, 30 (2011), arXiv:1004.2896 [astro-ph.HE].

[55] S. A. Balbus and J. F. Hawley, Reviews of Modern Physics 70, 1 (1998).

[56] M. Obergaulinger, P. Cerdá-Durán, E. Müller, and M. A. Aloy, A\&A 498, 241 (2009), arXiv:0811.1652.

[57] Y. Masada, T. Takiwaki, K. Kotake, and T. Sano, ApJ 759, 110 (2012), arXiv:1209.2360 [astro-ph.SR].

[58] S. E. Woosley and J. S. Bloom, ARA\&A 44, 507 (2006), arXiv:astro-ph/0609142.

[59] B. D. Metzger, D. Giannios, T. A. Thompson, N. Bucciantini, and E. Quataert, MNRAS 
413, 2031 (2011), arXiv:1012.0001 [astro-ph.HE],

[60] A. I. MacFadyen, S. E. Woosley, and A. Heger, ApJ 550, 410 (2001), arXiv:astro$\mathrm{ph} / 9910034$.

[61] S. Harikae, T. Takiwaki, and K. Kotake, ApJ 704, 354 (2009), arXiv:0905.2006 [astroph.HE].

[62] S. Harikae, K. Kotake, and T. Takiwaki, ApJ 713, 304 (2010), arXiv:0912.2590 [astroph.HE],

[63] P. Mészáros, Reports on Progress in Physics 69, 2259 (2006), arXiv:astro-ph/0605208.

[64] K. Kotake, Comptes Rendus Physique 14, 318 (2013), arXiv:1110.5107.

[65] H. Dimmelmeier, C. D. Ott, H.-T. Janka, A. Marek, and E. Müller, Physical Review Letters 98, 251101 (2007), arXiv:astro-ph/0702305.

[66] C. D. Ott, H. Dimmelmeier, A. Marek, H.-T. Janka, I. Hawke, B. Zink, and E. Schnetter, Physical Review Letters 98, 261101 (2007), arXiv:astro-ph/0609819.

[67] C. D. Ott, H. Dimmelmeier, A. Marek, H.-T. Janka, B. Zink, I. Hawke, and E. Schnetter, Classical and Quantum Gravity 24, 139 (2007), arXiv:astro-ph/0612638.

[68] A. Burrows and J. Hayes, Physical Review Letters 76, 352 (1996), arXiv:astro-ph/9511106.

[69] E. Mueller and H.-T. Janka, A\&A 317, 140 (1997).

[70] C. L. Fryer, ApJL 601, L175 (2004), arXiv:astro-ph/0312265.

[71] E. Müller, M. Rampp, R. Buras, H.-T. Janka, and D. H. Shoemaker, ApJ 603, 221 (2004), arXiv:astro-ph/0309833.

[72] A. Marek, H.-T. Janka, and E. Müller, A\&A 496, 475 (2009), arXiv:0808.4136.

[73] K. Kotake, N. Ohnishi, and S. Yamada, ApJ 655, 406 (2007), arXiv:astro-ph/0607224.

[74] K. Kotake, W. Iwakami, N. Ohnishi, and S. Yamada, ApJL 697, L133 (2009), arXiv:0904.4300 [astro-ph.HE],

[75] K. Kotake, W. Iwakami, N. Ohnishi, and S. Yamada, Astrophys. J. 704, 951 (2009), arXiv:0909.3622.

[76] J. W. Murphy, C. D. Ott, and A. Burrows, ApJ 707, 1173 (2009), arXiv:0907.4762 [astroph.SR],

[77] C. D. Ott, S. Ou, J. E. Tohline, and A. Burrows, ApJL 625, L119 (2005), arXiv:astro$\mathrm{ph} / 0503187$.

[78] S. Scheidegger, T. Fischer, S. C. Whitehouse, and M. Liebendörfer, A\&A 490, 231 (2008), 
arXiv:0709.0168.

[79] S. Scheidegger, R. Käppeli, S. C. Whitehouse, T. Fischer, and M. Liebendörfer, A\&A 514, A51 (2010).

[80] T. Kuroda, T. Takiwaki, and K. Kotake, Phys. Rev. D 89, 044011 (2014), arXiv:1304.4372 [astro-ph.HE],

[81] E. Müller, H.-T. Janka, and A. Wongwathanarat, A\&A 537, A63 (2012), arXiv:1106.6301 [astro-ph.SR].

[82] B. Müller, H.-T. Janka, and A. Marek, ApJ 766, 43 (2013), arXiv:1210.6984 [astro-ph.SR].

[83] C. D. Ott, A. Burrows, L. Dessart, and E. Livne, Physical Review Letters 96, 201102 (2006), arXiv:astro-ph/0605493.

[84] F. A. Rasio and S. L. Shapiro, Classical and Quantum Gravity 16, 1 (1999), gr-qc/9902019.

[85] P. R. Brady and S. Ray-Majumder, Classical and Quantum Gravity 21, 1839 (2004), grqc/0405036.

[86] T. Zwerger and E. Mueller, Astron. Astrophys. 320, 209 (1997).

[87] Classical and Quantum Gravity 26, 105005 (2009), arXiv:0810.5707 [gr-qc].

[88] C. Röver, M.-A. Bizouard, N. Christensen, H. Dimmelmeier, I. S. Heng, and R. Meyer, Phys. Rev. D 80, 102004 (2009), arXiv:0909.1093 [gr-qc].

[89] H. Dimmelmeier, C. D. Ott, A. Marek, and H. Janka, Phys. Rev. D 78, 064056 (2008), arXiv:0806.4953.

[90] J. Logue, C. D. Ott, I. S. Heng, P. Kalmus, and J. H. C. Scargill, Phys. Rev. D 86, 044023 (2012), arXiv:1202.3256 [gr-qc].

[91] W. J. Engels, R. Frey, and C. D. Ott, ArXiv e-prints (2014), arXiv:1406.1164 [gr-qc],

[92] Y. Gürsel and M. Tinto, Phys. Rev. D 40, 3884 (1989).

[93] M. Rakhmanov, Class. Quantum Grav. 23, 673 (2006).

[94] K. Hayama, S. D. Mohanty, M. Rakhmanov, and S. Desai, Classical and Quantum Gravity 24, 681 (2007).

[95] T. Z. Summerscales, A. Burrows, L. S. Finn, and C. D. Ott, ApJ 678, 1142 (2008), arXiv:0704.2157.

[96] C. D. Ott, A. Burrows, E. Livne, and R. Walder, Astrophys. J. 600, 834 (2004).

[97] K. Kotake, S. Yamada, K. Sato, K. Sumiyoshi, H. Ono, and H. Suzuki, Phys. Rev. D 69, 124004 (2004), arXiv:astro-ph/0401563. 
[98] E. Abdikamalov, S. Gossan, A. M. DeMaio, and C. D. Ott, ArXiv e-prints (2013), arXiv:1311.3678 [astro-ph.SR].

[99] J. Abadie, B. P. Abbott, R. Abbott, M. Abernathy, C. Adams, R. Adhikari, P. Ajith, B. Allen, G. Allen, E. Amador Ceron, and et al., Nuclear Instruments and Methods in Physics Research A 624, 223 (2010), arXiv:1007.3973 [gr-qc].

[100] K. Kotake, W. Iwakami Nakano, and N. Ohnishi, ArXiv e-prints (2011), arXiv:1106.0544 [astro-ph.HE],

[101] W. Iwakami, K. Kotake, N. Ohnishi, S. Yamada, and K. Sawada, Astrophys. J. 700, 232 (2009), arXiv:0811.0651.

[102] A. Heger, S. E. Woosley, and H. C. Spruit, Astrophys. J. 626, 350 (2005), arXiv:astro$\mathrm{ph} / 0409422$.

[103] E. Müller, H. . Janka, and A. Wongwathanarat, ArXiv e-prints (2011), arXiv:1106.6301 [astro-ph.SR].

[104] E. Müler and H.-T. Janka, Astron. Astrophys. 317, 140 (1997).

[105] E. Müller, M. Rampp, R. Buras, H.-T. Janka, and D. H. Shoemaker, Astrophys. J. 603, 221 (2004), arXiv:astro-ph/0309833.

[106] V. B. Braginskii and K. S. Thorne, Nature 327, 123 (1987).

[107] S. E. Woosley and T. A. Weaver, ApJS 101, 181 (1995).

[108] H. Dimmelmeier, J. A. Font, and E. Müller, A\&A 393, 523 (2002), arXiv:astro-ph/0204289.

[109] T. Kuroda, K. Kotake, and T. Takiwaki, ApJ 755, 11 (2012), arXiv:1202.2487 [astro-ph.HE],

[110] C. D. Ott, E. Abdikamalov, E. O'Connor, C. Reisswig, R. Haas, P. Kalmus, S. Drasco, A. Burrows, and E. Schnetter, Phys. Rev. D 86, 024026 (2012), arXiv:1204.0512 [astroph.HE].

[111] M. Obergaulinger, M. A. Aloy, H. Dimmelmeier, and E. Müller, Astron. Astrophys. 457, 209 (2006), arXiv:astro-ph/0602187.

[112] T. Takiwaki, K. Kotake, and K. Sato, Astrophys. J. 691, 1360 (2009), arXiv:0712.1949.

[113] M. Shibata, Y. T. Liu, S. L. Shapiro, and B. C. Stephens, Phys. Rev. D 74, 104026 (2006), arXiv:astro-ph/0610840.

[114] M. Obergaulinger, M. A. Aloy, and E. Müller, A\&A 450, 1107 (2006), arXiv:astro$\mathrm{ph} / 0510184$.

[115] S. e. a. Kawamura, Classical and Quantum Gravity 23, 125 (2006). 
[116] H. Kudoh, A. Taruya, T. Hiramatsu, and Y. Himemoto, Phys. Rev. D 73, 064006 (2006), arXiv:gr-qc/0511145.

[117] É. É. Flanagan and S. A. Hughes, Phys. Rev. D 57, 4566 (1998), arXiv:gr-qc/9710129.

[118] K. Thorne, in Three Hundred Years of Gravitation, edited by S. Hawking and W. Israel (Cambridge University Press, 1987) pp. 330-458.

[119] Http://gwcenter.icrr.u-tokyo.ac.jp/en/researcher/parameter.

[120] Y. Gürsel and M. Tinto, Phys. Rev. D 40, 3884 (1989).

[121] S. Klimenko, S. Mohanty, M. Rakhmanov, and G. Mitselmakher, Phys. Rev. D 72, 122002 (2005), gr-qc/0508068.

[122] S. D. Mohanty, Classical and Quantum Gravity 22, 1349 (2005).

[123] S. Klimenko, I. Yakushin, A. Mercer, and G. Mitselmakher, Classical and Quantum Gravity 25, 114029 (2008), arXiv:0802.3232 [gr-qc].

[124] P. J. Sutton, G. Jones, S. Chatterji, P. Kalmus, I. Leonor, S. Poprocki, J. Rollins, A. Searle, L. Stein, M. Tinto, and M. Was, New Journal of Physics 12, 053034 (2010), arXiv:0908.3665 $[\mathrm{gr}-\mathrm{qc}]$.

[125] K. Hayama and A. Nishizawa, Phys. Rev. D 87, 062003 (2013), arXiv:1208.4596 [gr-qc].

[126] A. Lazzarini and et al., LIGO technical document (1996).

[127] J. Makhoul, Proc. IEEE 63 (1975).

[128] S. Chatterji, L. Blackburn, G. Martin, and E. Katsavounidis, Class. Quantum Grav. 21, S1809 (2004).

[129] L. Wen and B. F. Schutz, Classical and Quantum Gravity 22, 1321 (2005), gr-qc/0508042.

[130] http://gwcenter.icrr.u-tokyo.ac.jp/en.

[131] S. Klimenko, G. Vedovato, M. Drago, G. Mazzolo, G. Mitselmakher, C. Pankow, G. Prodi, V. Re, F. Salemi, and I. Yakushin, Phys. Rev. D 83, 102001 (2011), arXiv:1101.5408 [astroph.IM].

[132] T. Yokozawa, M. Asano, T. Kayano, Y. Suwa, N. Kanda, Y. Koshio, and M. R. Vagins, ArXiv e-prints (2014), arXiv:1410.2050 [astro-ph.HE].

[133] I. Tamborra, G. Raffelt, F. Hanke, H.-T. Janka, and B. Müller, Phys. Rev. D 90, 045032 (2014), arXiv:1406.0006 [astro-ph.SR].

[134] K. Kotake, T. Takiwaki, Y. Suwa, W. Iwakami Nakano, S. Kawagoe, Y. Masada, and S.-i. Fujimoto, Advances in Astronomy 2012, 428757 (2012), 10.1155/2012/428757, arXiv:1204.2330 
[astro-ph.HE]. 